\title{
Regular graphs and the spectra of two-variable logic with counting
}

\author{
Eryk Kopczyński* Tony Tan ${ }^{\dagger}$
}

\begin{abstract}
The spectrum of a first-order logic sentence is the set of natural numbers that are cardinalities of its finite models. In this paper we show that when restricted to using only two variables, but allowing counting quantifiers, the spectra of first-order logic sentences are semilinear and hence, closed under complement. At the heart of our proof are semilinear characterisations for the existence of regular and biregular graphs, the class of graphs in which there are a priori bounds on the degrees of the vertices. Our proof also provides a simple characterisation of models of two-variable logic with counting - that is, up to renaming and extending the relation names, they are simply a collection of regular and biregular graphs.
\end{abstract}

Keywords: two-variable logic with counting, first-order spectra, regular graphs, semi-linear, Presburger arithmetic.

\section{Introduction}

The spectrum of a first-order sentence $\phi$, denoted by $\operatorname{SeEC}(\phi)$, is the set of natural numbers that are cardinalities of finite models of $\phi$. Or, more formally, $\operatorname{SPEC}(\phi)=\{n \mid$ there is a model of $\phi$ of size $n\}$. A set is a spectrum, if it is the spectrum of a first-order sentence.

In this paper we consider the logic $C^{2}$, the class of first-order sentences using only two variables and allowing counting quantifiers $\exists^{k} z \phi(z)$, where $k \geq 1$. Semantically $\exists^{k} z \phi(z)$ means there exist at least $k$ number of $z$ 's such that $\phi(z)$ holds. We prove that the spectra of $\mathrm{C}^{2}$ are precisely semilinear sets. In fact, our proof also shows that the family of models of a $\mathrm{C}^{2}$ formula can be viewed as a collection of regular graphs.

\section{Related works}

The notion of spectrum was introduced by Scholz in 34 . where he also asked whether there exists a necessary and sufficient condition for a set to be a spectrum. Since its publication, Scholz's question and many of its variants have been investigated by many researchers for the past 60 years. One of the arguably main open problems in this area is the one asked in [1, known as Asser's conjecture, whether the complement of a spectrum is also a spectrum.

The notion of spectrum has a deep connection with complexity theory as shown by Jones and Selman 21, as well as Fagin 4 independently that a set of integers is a spectrum if and only if its binary representation is in NE. Hence, Asser's conjecture is equivalent to asking whether $\mathrm{NE}=\mathrm{CO}-\mathrm{NE}$. It also immediately implies that if Asser's conjecture is false, i.e., there is a spectrum whose complement is not a spectrum, then $\mathrm{NP} \neq \mathrm{CO}-\mathrm{NP}$, hence $\mathrm{P} \neq \mathrm{NP}$. The converse implication is still open. An interesting result in 38 states that if spectra are precisely rudimentary sets, then $\mathrm{NE}=\mathrm{CO}-\mathrm{NE}$ and $\mathrm{NP} \neq \mathrm{CO}-\mathrm{NP}$ There are a number of interesting connections between spectrum and various models of computation such as RAM as well as intrinsic computational behavior. See,

\footnotetext{
*University of Warsaw, erykk@mimuw.edu.pl

$\dagger$ Hasselt University and Transnational University of Limburg, ptony.tan@gmail.com

* It should be noted that the class of rudimentary sets corresponds precisely to linear time hierarchy - the linear time analog of polynomial time hierarchy [39.
} 
for example, [8, 9, 10, 27, 33. We refer the reader to [2] for a more comprehensive treatment on the spectra problem and its history.

The logic $C^{2}$ is not the first logic known to have semilinear spectra. A well known Parikh theorem states the spectra of context-free languages are semilinear, and closed under complementation. Using the celebrated composition method, Gurevich and Shelah in [16 showed that the spectra of monadic second order logic with one unary function are semilinear. Compared to the work in [16, note that $\mathrm{C}^{2}$ one can express a few unary functions, hence our result does not follow from [16], and neither theirs from ours since we are restricted to using only two variables.

In 6 Fischer and Makowsky show that the many-sorted spectra of the monadic second-order logic with modulo counting over structures with bounded tree-width are semilinear. Intuitively, the many-sorted spectra of a formula are spectra which counts the cardinality of the unary predicates in the models of the formula, instead of just counting the sizes of the models. The semilinearity is obtained by reduction to regular tree languages and "pumping" argument. This result is orthogonal to ours, since structures expressible in $\mathrm{C}^{2}$ do not have bounded tree-width. An example is $d$-regular graphs for $d \geq 3$. Moreover, due to unbounded tree-width, it is very unlikely that one can apply some sort of "pumping" or automata theoretic argument as in [6] to obtain the semilinearity of $\mathrm{C}^{2}$ spectra.

As far as we know, $\mathrm{C}^{2}$ is the first logic known to have its spectra closed under complement without any restriction on the vocabulary nor in the interpretation. The result closest to ours is the one by É. Grandjean in [10] where he considers the spectra of first-order sentences using only one variable. A similar result due to M. Grohe and stated in [2], says that for every Turing machine $M$, there exists a first-order sentence $\phi_{M}$ using only three variables such that $\operatorname{SpEC}\left(\phi_{M}\right)=\left\{t^{2} \mid\right.$ $t$ is the length of an accepting run of $M\}$.

To end our study of related work, we should mention that the two-variable logic and many of its variants have been extensively studied, with the focus being mainly on the satisfiability problem. For more development in this direction, we refer the readers to $30,37,31,14,13,11,18,32,25,35]$ and the references therein.

\section{Sketch of our proof}

Consider the following instances of structures expressible in $\mathrm{C}^{2}$ 团

(Ex.1) $(c, d)$-biregular graphs: the bipartite graphs on the vertices $U \cup V$, where the degree of each vertex in $U$ and $V$ is $c$ and $d$, respectively.

(Ex.2) $(c, d)$-regular digraphs: the directed graphs in which the in-degree and the out-degree of each vertex is $c$ and $d$, respectively.

An observation from basic graph theory tells us that for "big enough" $M$ and $N$ t

(C1) there is a $(c, d)$-biregular graph in which $M$ vertices are of degree $c$ and $N$ vertices of degree $d$ if and only if $M c=N d$;

(C2) there is a $(c, d)$-regular digraph of $N$ vertices if and only if $N c=N d$, and hence, $c=d$.

These characterisations immediately imply that the spectra of the formulas (Ex.1) and (Ex.2) above are linear sets. It is from these observations that we draw our inspiration to prove the semilinearity of the spectra of $\mathrm{C}^{2}$.

More precisely, we show that given a $\mathrm{C}^{2}$ sentence $\varphi$, one can construct a Presburger formula $\psi$ that expresses precisely the spectrum of $\varphi$. Presburger formulas are first-order formulas with the relation symbols + and $\leq$ and constants 0 and 1 interpreted over the domain $\mathbb{N}$ in the natural way. It is shown by Ginsburg and Spanier in 7 that Presburger formulas express precisely the class of semilinear sets. That is, if $\psi(\bar{X})$ is a Presburger formula with free variables $\bar{X}=\left(X_{1}, \ldots, X_{k}\right)$, the set $\left\{\bar{N} \in \mathbb{N}^{k} \mid \varphi(\bar{N})\right.$ holds $\}$ is semilinear.

\footnotetext{
${ }^{\dagger}$ Though the result in this paper holds for arbitrary structures, it helps to assume that the structures of $\mathrm{C}^{2}$ are graphs in which the vertices and the edges are labelled with a finite number of colours.

$\ddagger$ "Big enough" means $M$ and $N$ are greater than a constant $K$ which depends only on $c$ and $d$.
} 
The crux of our construction of the Presburger formula is a generalisation of the characterisations (C1) $-(\mathrm{C} 2)$ above to the following setting. Let $\mathcal{C}$ be a set of $\ell$-colors, denoted by $\mathrm{col}_{1}, \mathrm{col}_{2}, \ldots, \mathrm{col}_{\ell}$, and let $C$ and $D$ be $(\ell \times m)$ - and $(\ell \times n)$-matrices whose entries are all non-negative integers. We say that a bipartite graph $G=(U, V, E)$ is $(C, D)$-biregular, if we can color its edges with colors from $\mathcal{C}$ such that there is a partition $U=U_{1} \cup \cdots \cup U_{m}$ and $V=V_{1} \cup \cdots \cup V_{n}$ where

- for every integer $1 \leq i \leq m$, for every vertex $u \in U_{i}$, for every $1 \leq j \leq \ell$, the number of edges with color $\mathrm{col}_{j}$ adjacent to $u$ is precisely $C_{j, i}$; and

- for every integer $1 \leq i \leq n$, for every vertex $v \in V_{i}$, for every $1 \leq j \leq \ell$, the number of edges with color $\mathrm{col}_{j}$ adjacent to $v$ is precisely $D_{j, i}$.

Our setting also allows us to say that the number of edges with color $\operatorname{col}_{j}$ adjacent to $v$ is at least $D_{j, i}$. In Theorem 5.1 we effectively construct a Presburger formula that characterises the set $\{N \mid$ there is a $(C, D)$-biregular graph of $N$ vertices $\}$.

In a similar manner, we can define $(C, D)$-regular digraphs, where $C$ and $D$ control the number of incoming and outgoing edges of each vertex, respectively. Likewise, we obtain a similar Presburger formula that characterises the set $\{N \mid$ there is a $(C, D)$-regular digraph of $N$ vertices $\}$ \& We then proceed to observe that the relations in every model of a $C^{2}$ formula can be partitioned in such a way that every part forms a $(C, D)$-regular digraph, and every two parts a $(C, D)$-biregular graph. In a sense this shows that the models of $\mathrm{C}^{2}$ is simply a collection of regular graphs. Applying the Presburger formula that characterises the existence of these regular graphs, we obtain the semilinearity of the spectra of $\mathrm{C}^{2}$ formulae.

For the converse direction, it is not that difficult to show that every semilinear set is also a spectrum of a $C^{2}$ sentence. Since semilinear sets are closed under complement, this establishes the fact that the spectra of $\mathrm{C}^{2}$ are closed under complement. It can also be deduced immediately from our proof that the many-sorted spectra of $\mathrm{C}^{2}$ are also semilinear. Moreover, our result extends trivially to the class $\exists \mathrm{SOC}^{2}$, the class of sentences of the form: $\exists R_{1} \cdots \exists R_{m} \phi$, where $R_{1}, \ldots, R_{m}$ are second-order variables and $\phi$ is a $C^{2}$ formula. We simply regard $R_{1}, \ldots, R_{m}$ as part of the signature.

\section{Outline of the paper}

This paper is organised as follows. In Section 2 we review the logic $C^{2}$ and state our main result: Theorem 2.1 which states that every $C^{2}$ spectrum can be express in a Presburger formula. The proof of Theorem 2.1 is rather complex. So we present its outline in Section 3, before its details in Sections 4-8. Finally we conclude with a few observations and future directions in Section 9 .

\section{The logic $C^{2}$}

In this section we review the definition of $C^{2}$ and mention the main result in this paper and its corollaries. We fix $\mathcal{P}=\left\{P_{1}, P_{2}, \ldots\right\}$ to be the set of predicate symbols of arity 1 ; and $\mathcal{R}=$ $\left\{R_{1}, R_{2}, \ldots\right\}$ the set of predicate symbols of arity 2 . Two-variable logic with counting, denoted by $\mathrm{C}^{2}$, is defined by the following syntax.

$$
\phi \quad::=\quad z=z|R(z, z)| P(z)|\neg \phi| \phi \wedge \phi \mid \exists^{k} z \phi
$$

where the variable $z$ ranges over $x, y$, and the symbols $R$ and $P$ over $\mathcal{R}$ and $\mathcal{P}$, respectively.

The quantifier $\exists^{k} z \phi$ means there are at least $k$ elements $z$ such that $\phi$ holds. Note that $\exists^{1} z \phi$ is the standard $\exists z \phi$, and $\forall z \phi$ is equivalent to $\neg \exists^{1} z \neg \phi$. By default, we assume that $\exists^{0} z \phi$ always holds.

$\S$ Closely related to our result is the work by S. L. Hakimi 17] which deals with the question: given a vector $\left(d_{1}, \ldots, d_{m}\right)$, is there a graph with vertices $v_{1}, \ldots, v_{m}$ whose degrees are precisely $d_{1}, \ldots, d_{m}$, respectively? Another related result concerns the notion of score sequence obtained by H. G. Landau 22] which deals with the question: given a vector $\left(d_{1}, \ldots, d_{m}\right)$, is there a tournament with vertices $v_{1}, \ldots, v_{m}$ whose outdegrees are precisely $d_{1}, \ldots, d_{m}$, respectively? These questions are evidently different from our characterisations provided in Section 5 
As usual, we write $\mathfrak{A} \models \phi$ to denote that the structure $\mathfrak{A}$ is a model of $\phi$ and $\operatorname{SpEC}(\phi)$ to denote the spectrum of $\phi$. Theorem 2.1 below is the main result in this paper. Its proof spans over Sections 4-8.

Theorem 2.1 For every $\phi \in C^{2}$, there exists a Presburger formula PREB $(x)$ such that the set $\{n \mid \operatorname{PREB}(n)$ holds $\}=\operatorname{SPEC}(\phi)$. Moreover, the formula PREB $(x)$ can be constructed effectively.

We should remark that Theorem 2.1 also holds for arbitrary vocabulary. Since $C^{2}$ uses only two variables, relations of greater arity such as $R(x, y, x, x, y)$ can be viewed simply as unary or binary relations; so we can create new binary and unary relations for each possible combination, and easily verify whether the result is consistent.

An immediate consequence of Theorem 2.1 is the spectra of $C^{2}$ are semilinear.

Corollary 2.2 For every sentence $\phi \in \mathrm{C}^{2}$, the spectrum $\operatorname{SPEC}(\phi)$ is semilinear.

On the other hand, it is not that difficult to show that every semilinear set is a spectrum of a $\mathrm{C}^{2}$ sentence, as formally stated below.

Proposition 2.3 For every semilinear set $\Lambda \subseteq \mathbb{N}$, there exists a sentence $\phi \in \mathrm{C}^{2}$ such that $\operatorname{SpEC}(\phi)=\Lambda$.

Proof. For a linear set $\Gamma_{m, n}=\{m+i n \mid i=0,1,2, \ldots\}$, consider the vocabulary $\tau_{m, n}=$ $\left\{A, B_{0}, B_{1}, \ldots, B_{n-1}, E\right\}$, where $A, B_{0}, \ldots, B_{n-1}$ are unary and $E$ binary. Consider the $C^{2}$ sentence $\phi_{m, n}$ which states that $A \cup B_{0} \cup \cdots \cup B_{n-1}$ partition the whole universe, the predicate $A$ contains exactly $m$ elements, and for every $x$, if $B_{i}(x)$ holds,

- there is exactly one $y$ such that $x \neq y$ and $B_{i+1} \bmod n(y)$ and $E(x, y)$ holds;

- there is exactly one $y$ such that $x \neq y$ and $B_{i-1} \bmod n(y)$ and $E(y, x)$ holds.

It is straightforward that $\operatorname{SpEc}\left(\phi_{m, n}\right)=\Gamma_{m, n}$. For a semilinear set, we simply takes the finite union of such $\phi_{m, n}$. This completes our proof of Proposition 2.3.

Now, take Corollary 2.2. apply the fact that semilinear sets are closed under complement, and then Proposition 2.3, we obtain the following corollary.

Corollary 2.4 The spectra of $\mathrm{C}^{2}$ sentences are closed under complement within $\mathrm{C}^{2}$.

\section{The plan for the proof of Theorem 2.1}

As mentioned earlier, the proof of Theorem 2.1 is rather complex and spans over Sections 4-8. We give its outline here.

- In Section 4 we define the logic QMLC (Quantified Modal Logic with Counting), which for our purpose, will be easier to work with. In particular, we show that $C^{2}$ and QMLC are equivalent in terms of spectra.

- In Section 5 we define the class of biregular graphs and regular digraphs. The main theorems in this section are Theorems 5.2 and 5.4. Theorem 5.2 gives us the Presburger characterisations of the existence of biregular graphs, while Theorem 5.4 the same characterisations for the regular digraphs.

- Equipped with Theorems 5.2 and [5.4, we construct the formula $\operatorname{PREB}(x)$ as required in Theorem 2.1 in Section 6 .

- However, the proofs of Theorems 5.2 and 5.4 are themselves rather long and involved. So we postpone the proof of Theorem 5.2 until Section 7 . The proof of Theorem 5.4 is similar to Theorem 5.2 so we simply sketch it in Section 8.

Figure 1 illustrates the interdependence among Sections 4-8. 


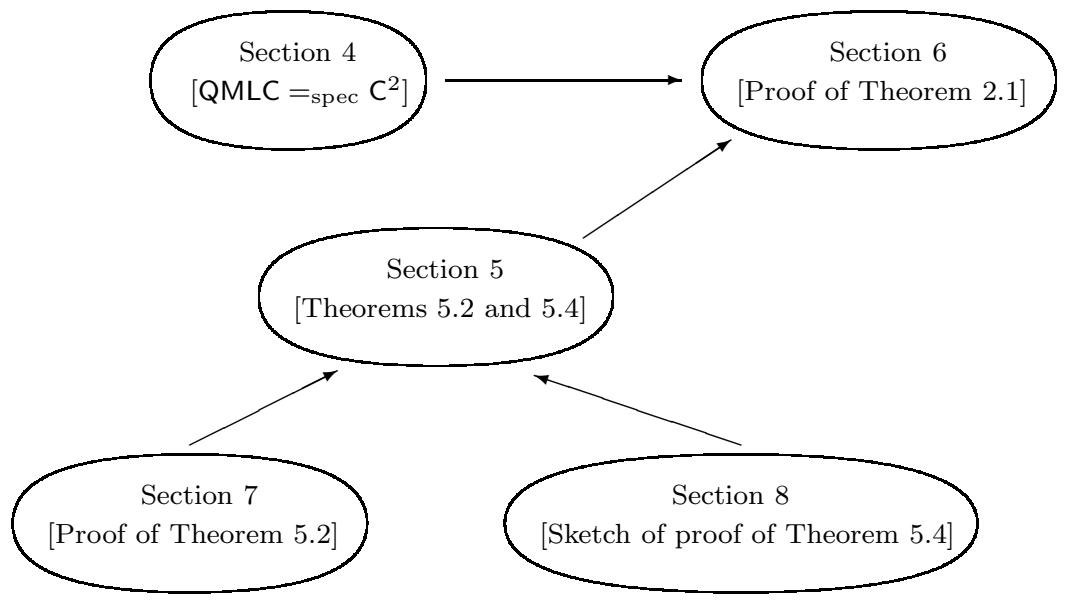

Figure 1: The skeleton for the proof of Theorem 2.1. Theorem 5.2 gives us the Presburger characterisations for the existence of biregular graphs, while Theorem 5.4 for the regular digraphs.

\section{Quantified modal logic with counting}

In this section we present quantified modal logic with counting (QMLC), which for our purpose, will be easier to work with. We are going to show that $C^{2}$ and QMLC are equivalent in terms of spectra. In fact, our proof shows that $C^{2}$ and QMLC are equivalent up to renaming/deleting/adding relational symbols, when QMLC are restricted to "complete" structures defined as follows. A structure $\mathfrak{A}$ is a complete structure, if it satisfies the following properties.

(N1) $\mathfrak{A}$ is a clique over $A$. That is, for every $a, b \in A$, either $a=b$ or $R(a, b)$ for some $R \in \mathcal{R}$.

(N2) Every binary relation in $\mathcal{R}$ does not intersect identity relation. That is, for every $R \in \mathcal{R}$, if $R(a, b)$ holds, $a \neq b$.

(N3) $\mathcal{R}$ is closed under inverse. That is, for every $R \in \mathcal{R}$, there exists $\overleftarrow{R} \in \mathcal{R}$ such that $\overleftarrow{R} \neq R$ and for every $a, b \in A, R(a, b)$ if and only if $\overleftarrow{R}(b, a)$.

(N4) The binary predicates in $\mathcal{R}$ are pairwise disjoint.

Our proof is an adaptation of the proof in [28, which shows that similar equivalence holds between two-variable logic and modal logic.

The class MLC of modal logic with counting is defined with the following syntax.

$$
\phi \quad:=\neg \phi|\alpha| \phi \wedge \phi \mid \diamond_{\beta}^{k} \phi
$$

where $\alpha$ ranges over $\mathcal{P}$ and $\beta$ over $\mathcal{R}$.

The semantics of MLC is as follows. Let $\mathfrak{A}$ be a structure of $\tau$ and $a \in A$ and $\phi$ be an MLC formula. That $\mathfrak{A}$ satisfies $\phi$ from $a$, denoted by $\mathfrak{A}, a \models \phi$, is defined as follows.

- $\mathfrak{A}, a \models P$, where $P \in \mathcal{P}$, if $P(a)$ holds in $\mathfrak{A}$.

- $\mathfrak{A}, a \models \neg \phi$, if $\mathfrak{A}, a \not \models \phi$.

- $\mathfrak{A}, a \models \phi_{1} \wedge \phi_{2}$, if $\mathfrak{A}, a \models \phi_{1}$ and $\mathfrak{A}, a \models \phi_{2}$.

- $\mathfrak{A}, a \models \diamond_{R}^{k} \phi$, if there exist at least $k$ elements $b_{1}, \ldots, b_{k} \in A$ such that $R\left(a, b_{i}\right)$ holds in $\mathfrak{A}$ and $\mathfrak{A}, b_{i} \models \phi$ for $i=1, \ldots, k$.

We define the class of quantified modal logic with counting, denoted by QMLC with the following syntax.

$$
\psi::=\quad \neg \psi\left|\psi_{1} \wedge \psi_{2}\right| \exists^{k} \phi
$$


where the formula $\phi \in$ MLC. A QMLC formula $\psi$ is called a basic QMLC, if it is of the form $\exists^{k} \phi$, where $\phi \in$ MLC.

The semantics of QMLC is as follows. Let $\mathfrak{A}$ be a structure of $\tau$ and $\psi \in$ QMLC. That $\mathfrak{A}$ satisfies $\psi$, denoted by $\mathfrak{A}=\psi$, is defined as follows.

- $\mathfrak{A} \models \neg \psi$, if it is not the case that $\mathfrak{A} \models \psi$.

- $\mathfrak{A} \models \psi_{1} \wedge \psi_{2}$, if $\mathfrak{A} \models \psi_{1}$ and $\mathfrak{A} \models \psi_{2}$.

- $\mathfrak{A} \models \exists^{k} \phi$, if there exist at least $k$ elements $a_{1}, \ldots, a_{k} \in A$ such that $\mathfrak{A}, a_{i} \models \phi$ for $i=1, \ldots, k$.

We denote by $\operatorname{SPEC}(\psi)$ the set consists of the size of complete structures of $\psi$. That is, $\operatorname{SpEC}(\psi)=$ $\{n \mid$ there is a complete structure $\mathfrak{A} \models \psi$ of size $n\}$. Note that for QMLC, the notion of spectrum is restricted to complete structures.

In the following we are going to show that from spectral point of view, $\mathrm{C}^{2}$ and QMLC are equivalent. The intuitive explanation for the requirement of complete structure is as follows. Notice that in QMLC we cannot express the negation of a binary relation $\neg R(x, y)$. Rather, to "express" $\neg R(x, y)$ in QMLC, we introduce a new relation symbol to capture $\neg R(x, y)$, hence, the requirement (N1) and (N4) in the complete structure. Similarly, in QMLC from an element $x$, we cannot express the "inverse" direction $R(y, x)$. So we introduce a new relation $\overleftarrow{R}$ that captures the "inverse" of $R$, and $R(y, x)$ will be simulated by $\overleftarrow{R}(x, y)$, instead, hence the requirement (N3). We require (N2) simply for technical convenience.

Theorem 4.1 below states formally the spectral equivalence between $C^{2}$ and QMLC, when QMLC is restricted to complete structures.

Theorem 4.1 For every $\varphi \in \mathrm{C}^{2}$, there is a QMLC formula $\psi$ such that

- for every structure $\mathfrak{A} \models \varphi$, there is a complete structure $\mathfrak{B} \models \psi$ where $|A|=|B|$;

- for every complete structure $\mathfrak{B} \models \psi$, there is a structure $\mathfrak{A} \models \varphi$ and $|A|=|B|$.

Proof. Let $\varphi \in \mathrm{C}^{2}$. By extending/renaming/deleting the relations, and by modifying the sentence $\varphi$, if necessary, we can obtain another $C^{2}$ sentence $\varphi^{\prime}$ such that

- for every structure $\mathfrak{A} \models \varphi$, there is a complete structure $\mathfrak{A}^{\prime} \models \varphi^{\prime}$ where $|A|=\left|A^{\prime}\right|$;

- for every complete structure $\mathfrak{A}^{\prime} \models \varphi^{\prime}$, there is a structure $\mathfrak{A} \models \varphi$ and $|A|=\left|A^{\prime}\right|$.

The details of the construction of $\varphi^{\prime}$ is straightforward, hence, omitted. For example, to achieve (N1) and (N4) we can introduce a new binary relation for each Boolean combination of relations in $\varphi$. We can do similar trick to achieve (N2) and (N3).

From this formula $\varphi^{\prime}$, we are going to construct the desired QMLC formula $\phi$. It consists of the following two steps.

1. Convert the sentence $\varphi^{\prime}$ into its "normal form" $\psi$ such that for every complete structure $\mathfrak{A}$, we have $\mathfrak{A} \models \varphi^{\prime}$ if and only if $\mathfrak{A} \models \psi$ 田

2. Convert the sentence $\psi$ into a "quantified modal logic" (QMLC) sentence $\phi$ such that for every complete structure $\mathfrak{A}$, we have $\mathfrak{A} \models \psi$ if and only if $\mathfrak{A} \models \phi$.

In the following paragraphs we are going to describe formally these two steps.

A $\mathrm{C}^{2}$ sentence is in normal form, if all the quantifiers are either of form

$$
\exists^{k} y(R(x, y) \wedge \theta(y)), \quad \text { or } \quad \exists^{k} x \theta(x)
$$

and all other applications of variables are of form $P(x)$, where $P$ is a unary predicate.

The $C^{2}$ sentence $\varphi^{\prime}$ can be converted into its equivalent sentence $\psi$ in normal form as follows.

\footnotetext{
`We would like to remark that the normal form here is different from the standard Scott's normal form.
} 
- First, we rewrite every subformula of the form $\exists^{k} y \theta(x, y)$ with one free variable $x$ into the following form:

$$
\begin{gathered}
\theta(x, x) \wedge \exists^{k-1} y((x \neq y) \wedge \theta(x, y)) \\
\vee \\
\exists^{k} y((x \neq y) \wedge \theta(x, y))
\end{gathered}
$$

After such rewriting, we can assume that every quantifier in $\varphi$ is of the form $\exists^{k} y((x \neq$ y) $\wedge \theta(x, y))$.

- Second, every quantification $\exists^{k} y((x \neq y) \wedge \theta(x, y))$, in which $\theta(x, y)$ contains a subformula $\alpha(x)$ depending only on $x$, can be rewritten into the form:

$$
\begin{gathered}
\neg \alpha(x) \wedge \exists^{k} y\left((x \neq y) \wedge \theta_{0}(x, y)\right) \\
\vee \\
\alpha(x) \wedge \exists^{k} y\left((x \neq y) \wedge \theta_{1}(x, y)\right)
\end{gathered}
$$

where $\theta_{0}(x, y)$ and $\theta_{1}(x, y)$ are obtained from $\theta$ by replacing $\alpha(x)$ with false and true, respectively. We can repeat this until $\theta(x, y)$ no longer has a subformula depending only on $x$.

After such rewriting we can assume that every quantifier in $\varphi$ is of the form

$$
\exists^{k} y((x \neq y) \wedge \theta(x, y)),
$$

where $\theta(x, y)$ does not contain any subformula depending only on $x$.

- Third, every quantification $\exists^{k} y((x \neq y) \wedge \theta(x, y))$ can be rewritten into the form:

$$
\bigvee_{f \in \Delta_{\mathcal{R}}^{k}} \bigwedge_{R \in \mathcal{R}} \exists^{f(R)} y\left(R(x, y) \wedge \theta_{R}(y)\right)
$$

where $\Delta_{\mathcal{R}}^{k}$ is the set of all functions $f: \mathcal{R} \rightarrow \mathbb{N}$ such that $\sum_{R \in \mathcal{R}} f(R)=k$, and $\theta_{R}(y)$ is obtained from $\theta(x, y)$ by replacing each $R^{\prime}(x, y)$ with true if $R=R^{\prime}$, and false otherwise.

By performing these three steps, we get the $\mathrm{C}^{2}$ sentence $\psi$ in the normal form. Particularly, for every complete structure $\mathfrak{A}$, we have $\mathfrak{A} \models \varphi^{\prime}$ if and only if $\mathfrak{A} \models \psi$.

Now from this $C^{2}$ sentence $\psi$ in normal form, the construction of its QMLC sentence $\phi=F(\psi)$ can be done inductively as follows. There are two cases.

1. $\vartheta$ has no free variable.

- If $\vartheta$ is $\neg \vartheta_{1}$, then $F(\vartheta)=\neg F\left(\vartheta_{1}\right)$.

- If $\vartheta$ is $\vartheta_{1} \wedge \vartheta_{2}$, then $F(\vartheta)=F\left(\vartheta_{1}\right) \wedge F\left(\vartheta_{2}\right)$.

- If $\vartheta$ is $\exists^{k} x \vartheta_{1}(x)$, then $F(\vartheta)=\exists^{k} F\left(\vartheta_{1}(x)\right)$.

2. $\vartheta$ has one free variable $x$.

- If $\vartheta(x)$ is $P(x)$, then $F(\vartheta(x))=P$.

- If $\vartheta(x)$ is $\vartheta_{1}(x) \wedge \vartheta_{2}(x)$, then $F(\vartheta(x))=F\left(\vartheta_{1}(x)\right) \wedge F\left(\vartheta_{2}(x)\right)$.

- If $\vartheta(x)$ is $\neg \vartheta_{1}(x)$, then $F(\vartheta(x))=\neg F\left(\vartheta_{1}(x)\right)$.

- If $\vartheta(x)$ is $\exists^{k} y R(x, y) \wedge \vartheta_{1}(x, y)$, then $F(\vartheta(x))=\diamond_{R}^{k} F\left(\vartheta_{1}(x, y)\right)$.

The case when $\vartheta$ has one free variable $y$ can be handled in a symmetrical way.

By a straightforward induction, we can show that for every compete structure $\mathfrak{A}, \mathfrak{A} \models \vartheta$ if and only if $\mathfrak{A} \models F(\vartheta)$. In particular, from the equivalences between $\varphi$ and $\varphi^{\prime}$, between $\varphi^{\prime}$ and $\psi$ as well as between $\psi$ and $\phi=F(\psi)$, we obtain that

- for every structure $\mathfrak{A} \models \varphi$, there is a complete structure $\mathfrak{B} \models \psi$ such that $|A|=|B|$;

- for every complete structure $\mathfrak{B} \models \psi$, there is a structure $\mathfrak{A} \models \varphi$ such that $|A|=|B|$.

This concludes our proof of Theorem 4.1 


\section{Regular graphs}

In this section we are going to introduce two types of regular graphs: biregular graphs (bipartite regular graphs) and regular digraphs. The main results in this section are Theorems 5.2 and 5.4 , which will be used in our proof of Theorem [2.1. For the sake of readability, we postpone their proofs until Sections 7 and 8 .

\subsection{Biregular graphs}

An $\ell$-type bipartite graph is $G=\left(U, V, E_{1}, \ldots, E_{\ell}\right)$, where $E_{1}, \ldots, E_{\ell}$ are pairwise disjoint subsets of $U \times V$. Elements in $E_{i}$ are called $E_{i}$-edges. It helps to think of $G$ as a bipartite graph in which the edges are coloured with $\ell$ number of colours.

For a vertex $u \in U \cup V$, $\operatorname{deg}_{E_{i}}(u)$ denotes the number of $E_{i}$-edges adjacent to it, and $\operatorname{deg}(u)=$ $\sum_{i=1}^{\ell} \operatorname{deg}_{E_{i}}(u)$. We write $\operatorname{deg}(G)=\max \{\operatorname{deg}(u) \mid u$ is a vertex in $G\}$. For an integer $d \in \mathbb{N}$, we write $\operatorname{deg}_{E_{i}}(u)=d$, to denote $\operatorname{deg}_{E_{i}}(u) \geq d$.

Let $\mathbb{N}$ denote the set of natural numbers $\{0,1,2, \ldots\}$ and $\bullet \mathbb{N}=\left\{\bullet_{0}, \nabla^{\prime}, \ldots\right\}$ and $\mathbb{B}=\mathbb{N} \cup \mathbb{N}$. We write $\mathbb{B}^{\ell \times m}$ to denote the set of $\ell \times m$ matrices whose entries are from $\mathbb{B}$. The entry in row $i$ and column $j$ of a matrix $D \in \mathbb{B}^{\ell \times m}$ is denoted by $D_{i, j}$.

Let $C \in \mathbb{B}^{\ell \times m}$ and $D \in \mathbb{B}^{\ell \times n}$. An $\ell$-type bipartite graph $G=\left(U, V, E_{1}, \ldots, E_{\ell}\right)$ is $(C, D)$ biregular, if there is a partition $U=U_{1} \cup \cdots \cup U_{m}$ and $V=V_{1} \cup \cdots \cup V_{n}$ such that the following holds.

- For every $i=1, \ldots, \ell$, for every $j=1, \ldots, m$, for every vertex $u \in U_{j}, \operatorname{deg}_{E_{i}}(u)=C_{i, j}$.

- For every $i=1, \ldots, \ell$, for every $j=1, \ldots, n$, for every vertex $v \in V_{j}, \operatorname{deg}_{E_{i}}(v)=D_{i, j}$.

We call the partitions $U=U_{1} \cup \cdots \cup U_{m}$ and $V=V_{1} \cup \cdots \cup V_{n}$ the witness of the $(C, D)$-biregularity of $G$. We say that the $(C, D)$-biregular graph $G$ is of size $(\bar{M}, \bar{N})$, if $\bar{M}=\left(\left|U_{1}\right|, \ldots,\left|U_{m}\right|\right)$ and $\bar{N}=\left(\left|V_{1}\right|, \ldots,\left|V_{n}\right|\right)$.

Theorem 5.1 For every two matrices $C \in \mathbb{B}^{\ell \times m}$ and $D \in \mathbb{B}^{\ell \times n}$, there is a Presburger formula $\operatorname{BiRE}_{C, D}(\bar{X}, \bar{Y})$, where $\bar{X}=\left(X_{1}, \ldots, X_{m}\right)$ and $\bar{Y}=\left(Y_{1}, \ldots, Y_{n}\right)$ such that the following holds. There exists an $\ell$-type $(C, D)$-biregular graph of size $(\bar{M}, \bar{N})$ if and only if $\operatorname{BiRE}_{C, D}(\bar{M}, \bar{N})$ holds.

Theorem 5.1 is then generalised to the case of complete bipartite graphs. An $\ell$-type bipartite graph $G=\left(U, V, E_{1}, \ldots, E_{\ell}\right)$ is complete, if $U \times V=E_{1} \cup \cdots \cup E_{\ell}$. If $G$ is also a $(C, D)$-biregular graph, then we call it a $(C, D)$-complete-biregular graph.

The following theorem is the main result in this subsection that will be used in the proof in Section 6.

Theorem 5.2 For every two matrices $C \in \mathbb{B}^{\ell \times m}$ and $D \in \mathbb{B}^{\ell \times n}$, there is a Presburger formula COMP-BiREG $G_{C D}(\bar{X}, \bar{Y})$, where $\bar{X}=\left(X_{1}, \ldots, X_{m}\right)$ and $\bar{Y}=\left(Y_{1}, \ldots, Y_{n}\right)$ such that the following holds. There exists a $(C, D)$-complete-biregular graph of size $(\bar{M}, \bar{N})$ if and only if COMP-BiREG $C, D(\bar{M}, \bar{N})$ holds.

\subsection{Regular digraphs}

An $\ell$-type directed graph (or, digraph for short) is a tuple $G=\left(V, E_{1}, \ldots, E_{\ell}\right)$, where $E_{1}, \ldots, E_{\ell}$ are pairwise disjoint irreflexive relations on $V$ and for every $u, v \in V$, if $(u, v) \in E_{1} \cup \cdots \cup E_{\ell}$, then the inverse direction $(v, u) \notin E_{1} \cup \cdots \cup E_{\ell}$. Edges in $E_{i}$ are called $E_{i}$-edges.

We will write in- $\operatorname{deg}_{E_{i}}(u)$ to denote the number of incoming $E_{i}$-edges toward the vertex $u$, and out-deg $E_{i}(u)$ the number of outgoing $E_{i}$-edges from the vertex $u$. As before, for an integer $d \in \mathbb{N}$, we write in- $\operatorname{deg}_{E_{i}}(u)=d$ and out-deg $E_{E_{i}}(u)=d$, to indicate that in-deg $E_{i}(u) \geq d$ and in- $\operatorname{deg}_{E_{i}}(u) \geq d$, respectively.

Let $C, D \in \mathbb{B}^{\ell \times m}$. An $\ell$-type digraph $G=\left(V, E_{1}, \ldots, E_{\ell}\right)$ is $(C, D)$-regular-digraph, if there exists a partition $V=V_{1} \cup \cdots \cup V_{m}$ such that for each $i=1, \ldots, \ell$, for each $j=1, \ldots, m$, for each vertex $v \in V_{j}$, in- $\operatorname{deg}_{E_{i}}(v)=C_{i, j}$ and out- $\operatorname{deg}_{E_{i}}(v)=D_{i, j}$. We call $V_{1} \cup \cdots \cup V_{m}$ a witness 
of the $(C, D)$-regularity of $G$ and the graph $G$ is of size $\bar{N}=\left(N_{1}, \ldots, N_{m}\right)$, if $\left(N_{1}, \ldots, N_{m}\right)=$ $\left(\left|V_{1}\right|, \ldots,\left|V_{m}\right|\right)$.

Theorem 5.3 For every $C, D \in \mathbb{B}^{\ell \times m}$, there exists a Presburger formula $R E G_{C, D}(\bar{X})$, where $\bar{X}=$ $\left(X_{1}, \ldots, X_{m}\right)$ such that the following holds. There exists a $(C, D)$-regular-digraph of size $\bar{N}$ if and only if $R E G_{C, D}(\bar{N})$ holds.

Similar to Section 5.1 Theorem 5.3 will be generalised to the case of complete regular digraph. An $\ell$-type graph $G=\left(V, E_{1}, \ldots, E_{\ell}\right)$ is a complete digraph, if for every two different vertices $u, v$, either $(u, v)$ or $(v, u)$ is in $E_{1} \cup \cdots \cup E_{\ell}$. If $G$ is also a $(C, D)$-regular, then we call $G$ a $(C, D)$ complete-regular digraph.

The following theorem is the main result in this subsection that will be used in the proof in Section 6 .

Theorem 5.4 For every $C, D \in \mathbb{B}^{\ell \times m}$, there exists a Presburger formula $\operatorname{COMP} R E G_{C, D}(\bar{X})$, where $\bar{X}=\left(X_{1}, \ldots, X_{m}\right)$ such that the following holds. There exists a $(C, D)$-complete-regular digraph of size $\bar{N}$ if and only if COMP-REG $G_{C, D}(\bar{N})$ holds.

\section{Proof of Theorem 2.1}

Now we are ready to prove Theorem 2.1. Let $\phi$ be a QMLC sentence. Recall that a basic QMLC formula is of the form $\exists^{k} \varphi$, where $\varphi \in$ MLC. We also assume that in $\phi$ we have "pushed" all the negations inside so that they are applied only to basic QMLC. We are going to construct a Presburger formula $\operatorname{PREB}_{\phi}(x)$ such that $\operatorname{SPEC}(\phi)=\left\{n \mid \operatorname{PREB}_{\phi}(n)\right.$ holds $\}$.

Before we proceed, we need a few auxiliary notations. Let $\mathcal{P}$ be the set of unary predicates used in $\phi$ and $\mathcal{R}=\left\{R_{1}, \ldots, R_{\ell}, \overleftarrow{R}_{1}, \ldots, \overleftarrow{R}_{\ell}\right\}$ the set of binary relations used in $\phi$, where $\overleftarrow{R}_{i}$ is the inverse relation of $R_{i}$. Let $K$ be the integer such that for all subformulae $\diamond_{R}^{l} \psi$ in $\phi$, we have $l \leq K$.

We denote by $\mathcal{M}_{\phi}$ the set of all MLC subformulae of $\phi$ and their negations. A type in $\phi$ is a subset $T \subseteq \mathcal{M}_{\phi}$ such that

- if $\varphi_{1} \wedge \varphi_{2} \in T$, then both $\varphi_{1}, \varphi_{2} \in T$;

- $\varphi \in T$ if and only if $\neg \varphi \notin T$;

- if $\neg\left(\varphi_{1} \wedge \varphi_{2}\right) \in T$, then at least one of $\neg \varphi_{1}, \neg \varphi_{2} \in T$.

For a structure $\mathfrak{A}$ (not necessarily a model of $\phi$ ) and an element $a \in A$, we define the type of a in $\mathfrak{A}$, denoted by type $\operatorname{\mathfrak {A}}_{\mathfrak{A}}(a) \subseteq \mathcal{M}_{\phi}$, where $\varphi \in \operatorname{type}_{\mathfrak{A}}(a)$ if and only if $\mathfrak{A}, a \models \varphi$. For a type $T$, we write $T(\mathfrak{A})$ to denote the set of elements in $A$ with type $T$. Note that the sets $T(\mathfrak{A})$ 's are pairwise disjoint. We let $\mathcal{T}_{\phi}$ to be the set of all types in $\phi$.

We say that a function $f: \mathcal{T}_{\phi} \times \mathcal{R} \times \mathcal{T}_{\phi} \rightarrow\{0,1, \ldots, K\} \cup\{\boldsymbol{\sim}\}$ is consistent, if for every $T \in \mathcal{T}_{\phi}$ the following holds.

- If $\diamond_{R}^{l} \mu \in T$, then $\sum_{T^{\prime} \text { s.t. } T^{\prime} \ni \mu} f\left(T, R, T^{\prime}\right) \geq l$.

- If $\neg\left(\diamond_{R}^{l} \mu\right) \in T$, then $\sum_{T^{\prime} \text { s.t. } T^{\prime} \ni \mu} f\left(T, R, T^{\prime}\right) \leq l-1$, and $f\left(T, R, T^{\prime}\right) \in \mathbb{N}$, for every $R \in \mathcal{R}$ and for every type $T^{\prime} \ni \mu$.

In the following we enumerate the set of all consistent functions $\mathcal{F}=\left\{f_{1}, \ldots, f_{m}\right\}$, the set of all types in $\mathcal{T}_{\phi}=\left\{T_{1}, \ldots, T_{n}\right\}$, and the set $\mathcal{T}_{\phi} \times \mathcal{F}=\left\{\left(T_{1}, f_{1}\right), \ldots,\left(T_{n}, f_{m}\right)\right\}$.

The desired Presburger formula $\operatorname{PREB}_{\phi}(x)$ is defined as the formula:

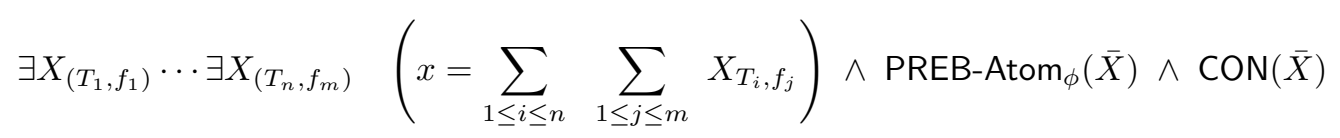

where $\bar{X}=\left(X_{\left(T_{1}, f_{1}\right)}, \ldots, X_{\left(T_{n}, f_{m}\right)}\right)$ is the vector of all the variables $X_{(T, f)}$ 's.

The intended meaning of the variable $X_{T_{i}, f_{j}}$ and the formulas PREB-Atom ${ }_{\phi}(x)$ and $\operatorname{CON}(\bar{X})$ is as follows. The variable $X_{T_{i}, f_{j}}$ is to represent the number of elements of type $T_{i}$ and for each 
binary relation $R \in \mathcal{R}$ and a type $S \in \mathcal{T}_{\phi}$, there is $f\left(T_{i}, R, S\right)$ number of outgoing $R$-edges towards the elements of type $S$.

Naturally, the total number of all elements in the universe will be the sum of all $X_{T_{i}, f_{j}}$ 's, hence, the sum:

$$
x=\sum_{1 \leq i \leq n} \sum_{1 \leq j \leq m} X_{T_{i}, f_{j}} .
$$

The formula PREB-Atom ${ }_{\phi}(x)$ is to make sure that the satisfiability of the QMLC sentence is preserved. Formally, it is defined inductively as follows. (Recall that all the negations have been "pushed" inside so that they are applied only to basic QMLC.)

- If $\phi:=\exists^{k} \varphi$, then PREB-Atom $\phi:=\sum_{(T, f) \text { s.t. } \varphi \in T} X_{(T, f)} \geq k$.

- If $\phi:=\neg \exists^{k} \varphi$, then PREB-Atom $\phi:=\sum_{(T, f) \text { s.t. } \varphi \in T} X_{(T, f)} \leq k-1$.

- If $\phi:=\phi_{1} \wedge \phi_{2}$, then PREB-Atom ${ }_{\phi}:=$ PREB-Atom $_{\phi_{1}} \wedge$ PREB-Atom $\phi_{\phi_{2}}$.

- If $\phi:=\phi_{1} \vee \phi_{2}$, then PREB-Atom ${ }_{\phi}:=$ PREB-Atom $_{\phi_{1}} \vee$ PREB-Atom $\phi_{\phi_{2}}$.

Finally, the formula $\operatorname{CON}(\bar{X})$ is to makes sure that the solution to each variable $X_{T_{i}, f_{j}}$ is "consistent" to the intended meaning of the type $T_{i}$ and function $f_{j}$. That is, for every types $S, T \in \mathcal{T}_{\phi}$ the following holds.

- Every solution $\bar{M}_{T}$ to the variables $\bar{X}_{T}=\left(X_{T, f_{1}}, \ldots, X_{T, f_{m}}\right)$ corresponds to a $\left(D_{T}, \overleftarrow{D}_{T}\right)$ complete-regular digraph of size $\bar{M}_{T}$, where the matrices $D_{T}, \overleftarrow{D}_{T} \in \mathbb{B}^{\ell \times m}$ are as follows.

$$
D_{T}:=\left(\begin{array}{cccc}
f_{1}\left(T, R_{1}, T\right) & f_{2}\left(T, R_{1}, T\right) & \cdots & f_{m}\left(T, R_{1}, T\right) \\
f_{1}\left(T, R_{2}, T\right) & f_{2}\left(T, R_{2}, T\right) & \cdots & f_{m}\left(T, R_{2}, T\right) \\
\vdots & \vdots & \ddots & \vdots \\
f_{1}\left(T, R_{\ell}, T\right) & f_{2}\left(T, R_{\ell}, T\right) & \cdots & f_{m}\left(T, R_{\ell}, T\right)
\end{array}\right)
$$

and

$$
\overleftarrow{D}_{T}:=\left(\begin{array}{cccc}
f_{1}\left(T, \overleftarrow{R}_{1}, T\right) & f_{2}\left(T, \overleftarrow{R}_{1}, T\right) & \cdots & f_{m}\left(T, \overleftarrow{R}_{1}, T\right) \\
f_{1}\left(T, \overleftarrow{R}_{2}, T\right) & f_{2}\left(T, \overleftarrow{R}_{2}, T\right) & \cdots & f_{m}\left(T, \overleftarrow{R}_{2}, T\right) \\
\vdots & \vdots & \ddots & \vdots \\
f_{1}\left(T, \overleftarrow{R}_{\ell}, T\right) & f_{2}\left(T, \overleftarrow{R}_{\ell}, T\right) & \cdots & f_{m}\left(T, \overleftarrow{R}_{\ell}, T\right)
\end{array}\right)
$$

Notice that the matrix $D_{T}$ contains only the information on the degree of $R_{1}, \ldots, R_{\ell}$, while $\overleftarrow{D}_{T}$ the information on the degree of $\overleftarrow{R}_{1}, \ldots, \overleftarrow{R}_{\ell}$. This is because the incoming $R_{i}$ edges to an element $v$ are precisely the outgoing $\overleftarrow{R}_{i}$ edges from $v$, and vice versa, the incoming $\overleftarrow{R}_{i}$ edges from an element $v$ are precisely the outgoing $R_{i}$ edges to $v$.

- Every solution $\bar{M}_{S}, \bar{M}_{T}$ to the variables $\bar{X}_{S}=\left(X_{S, f_{1}}, \ldots, X_{S, f_{m}}\right)$ and $\bar{X}_{T}=\left(X_{T, f_{1}}, \ldots, X_{T, f_{m}}\right)$ corresponds to a $\left(D_{S \rightarrow T}, \overleftarrow{D}_{S \rightarrow T}\right)$-complete-biregular digraph of size $\left(\bar{M}_{S}, \bar{M}_{T}\right)$, where the matrices $D_{S \rightarrow T}, \overleftarrow{D}_{S \rightarrow T} \in \mathbb{B}^{\ell \times m}$ are as follows.

$$
D_{S \rightarrow T}:=\left(\begin{array}{cccc}
f_{1}\left(S, R_{1}, T\right) & f_{2}\left(S, R_{1}, T\right) & \cdots & f_{m}\left(S, R_{1}, T\right) \\
f_{1}\left(S, R_{2}, T\right) & f_{2}\left(S, R_{2}, T\right) & \cdots & f_{m}\left(S, R_{2}, T\right) \\
\vdots & \vdots & \ddots & \vdots \\
f_{1}\left(S, R_{\ell}, T\right) & f_{2}\left(S, R_{\ell}, T\right) & \cdots & f_{m}\left(S, R_{\ell}, T\right) \\
f_{1}\left(S, \overleftarrow{R}_{1}, T\right) & f_{2}\left(S, \overleftarrow{R}_{1}, T\right) & \cdots & f_{m}\left(S, \overleftarrow{R}_{1}, T\right) \\
f_{1}\left(S, \overleftarrow{R}_{2}, T\right) & f_{2}\left(S, \overleftarrow{R}_{2}, T\right) & \cdots & f_{m}\left(S, \overleftarrow{R}_{2}, T\right) \\
\vdots & \vdots & \ddots & \vdots \\
f_{1}\left(S, \overleftarrow{R}_{\ell}, T\right) & f_{2}\left(S, \overleftarrow{R}_{\ell}, T\right) & \cdots & f_{m}\left(S, \overleftarrow{R}_{\ell}, T\right)
\end{array}\right)
$$


and

$$
\overleftarrow{D}_{S \rightarrow T}:=\left(\begin{array}{cccc}
f_{1}\left(T, \overleftarrow{R}_{1}, S\right) & f_{2}\left(T, \overleftarrow{R}_{1}, S\right) & \cdots & f_{m}\left(T, \overleftarrow{R}_{1}, S\right) \\
f_{1}\left(T, \overleftarrow{R}_{2}, S\right) & f_{2}\left(T, \overleftarrow{R}_{2}, S\right) & \cdots & f_{m}\left(T, \overleftarrow{R}_{2}, S\right) \\
\vdots & \vdots & \ddots & \vdots \\
f_{1}\left(T, \overleftarrow{R}_{\ell}, S\right) & f_{2}\left(T, \overleftarrow{R}_{\ell}, S\right) & \cdots & f_{m}\left(T, \overleftarrow{R}_{\ell}, S\right) \\
f_{1}\left(T, R_{1}, S\right) & f_{2}\left(T, R_{1}, S\right) & \cdots & f_{m}\left(T, R_{1}, S\right) \\
f_{1}\left(T, R_{2}, S\right) & f_{2}\left(T, R_{2}, S\right) & \cdots & f_{m}\left(T, R_{2}, S\right) \\
\vdots & \vdots & \ddots & \vdots \\
f_{1}\left(T, R_{\ell}, S\right) & f_{2}\left(T, R_{\ell}, S\right) & \cdots & f_{m}\left(T, R_{\ell}, S\right)
\end{array}\right)
$$

Notice that in the matrix $D_{S \rightarrow T}$ the first $\ell$ rows contains the information on the degree of $R_{1}, \ldots, R_{\ell}$, and the last $\ell$ rows the information on the degree of $\overleftarrow{R}_{1}, \ldots, \overleftarrow{R}_{\ell}$ from the type $S$ to the type $T$; while in the matrix $\bar{D}_{S \rightarrow T}$ it is the opposite and the direction is from the type $T$ to the type $S$. Similar as in the $D_{T}, \bar{D}_{t}$ case above, this is because the incoming $R_{i}$ edges to an element $v$ are precisely the outgoing $\overleftarrow{R}_{i}$ edges from $v$, and vice versa, the outgoing $R_{i}$ edges from an element $v$ are precisely the incoming $\overleftarrow{R}_{i}$ edges to $v$.

Now the formula $\operatorname{CON}(\bar{X})$ is simply the conjunction:

$$
\begin{aligned}
\operatorname{CON}(\bar{X}):= & \bigwedge_{1 \leq i \leq n} \operatorname{COMP}_{1 \leq n E D_{D_{i}}, \overleftarrow{D}_{T_{i}}}\left(\bar{X}_{T}\right) \\
& \wedge \bigwedge_{1 \leq j<i \leq n} \operatorname{COMP} \operatorname{BiREG}_{D_{T_{i} \rightarrow T_{j}}, \overleftarrow{D}_{T_{i} \rightarrow T_{j}}}\left(\bar{X}_{T_{i}}, \bar{X}_{T_{j}}\right)
\end{aligned}
$$

where $\bar{X}_{T_{i}}=\left(X_{\left(T_{i}, f_{1}\right)}, \ldots, X_{\left(T_{i}, f_{m}\right)}\right)$ is the vector of variables associated with the type $T_{i}$.

We are going to show that $\mathrm{PREB}_{\phi}$ defines precisely the spectrum of $\phi$, as stated in the claim below. Abusing the notation, we let $\operatorname{PREB}_{\phi}$ itself to denote the set $\left\{n \mid \operatorname{PREB}_{\phi}(n)\right.$ holds $\}$. Recall also that as defined in Section 4, the spectrum of a QMLC sentence $\phi$ is restricted to the complete structures.

Claim 1 For every $\mathrm{QMLC}$ sentence $\phi, P R E B_{\phi}=\operatorname{spec}(\phi)$, where $P R E B_{\phi}(x)$ is the formula

$$
\exists X_{\left(T_{1}, f_{1}\right)} \cdots \exists X_{\left(T_{n}, f_{m}\right)} \quad\left(x=\sum_{1 \leq i \leq n} \sum_{1 \leq j \leq m} X_{T_{i}, f_{j}}\right) \wedge \operatorname{PREB-Atom}(\bar{X}) \wedge \operatorname{CON}(\bar{X})
$$

and $\bar{X}=\left(X_{\left(T_{1}, f_{1}\right)}, \ldots, X_{\left(T_{n}, f_{m}\right)}\right)$ is the vector of all the variables $X_{(T, f)}$ 's.

The proof is by induction on $\phi$. The base case is when $\phi$ is a basic QMLC sentence or the negation of a basic QMLC sentence. We consider first the case when $\phi$ is a basic QMLC formula of the form $\exists^{k} \varphi$, where $\varphi \in \mathrm{MLC}$. In this case PREB-Atom ${ }_{\phi}(\bar{X})$ is $\sum_{(T, f) \text { s.t. } \varphi \in T} X_{(T, f)} \geq k$.

We first show the direction $\mathrm{PREB}_{\phi} \subseteq \operatorname{spec}(\phi)$. Let $N \in \mathrm{PREB}_{\phi}$. Let $\bar{M}=\left(M_{T_{1}, f_{1}}, \ldots, M_{T_{n}, f_{m}}\right)$ be the witnesses to $\bar{X}$ such that $\operatorname{PREB}_{\phi}(N)$ holds. In the following we are going to write $\bar{M}_{T}$ to denote $\left(M_{T, f_{1}}, \ldots, M_{T, f_{m}}\right)$ for every type $T \in \mathcal{T}_{\phi}$.

Since $x=\sum_{(T, f)} X_{T, f}$, we have $N=\sum_{(T, f)} M_{(T, f)}$. We take a set $V$ of $N$ vertices and we partition $V=V_{\left(T_{1}, f_{1}\right)} \cup \cdots \cup V_{\left(T_{n}, f_{m}\right)}$ such that $\left|V_{(T, f)}\right|=M_{(T, f)}$ for each $T \in \mathcal{T}_{\phi}$ and $f \in \mathcal{F}$. We denote by $V_{T}=V_{\left(T, f_{1}\right)} \cup \cdots \cup V_{\left(T, f_{m}\right)}$ for each $T \in \mathcal{T}_{\phi}$.

Since $\operatorname{CON}(\bar{M})$ holds, by Theorems 5.4, for each $T \in \mathcal{T}_{\phi}$, there exists a $\left(D_{T}, \overleftarrow{D}_{T}\right)$-completeregular digraph $G_{T}=\left(V_{T}, R_{T, 1}, \ldots, R_{T, \ell}\right)$ of size $\bar{M}_{T}$, with $V_{T}=V_{\left(T, f_{1}\right)} \cup \cdots \cup V_{\left(T, f_{m}\right)}$ be the witness of the $\left(D_{T}, \overleftarrow{D}_{T}\right)$-regularity. This means that for every vertex $v \in V_{T, f_{i}}$, for every $R \in\left\{R_{1}, \ldots, R_{\ell}\right\}$,

- out-deg ${ }_{R}(v)$ in the graph $G_{T}$ is $f_{i}(T, R, T)$;

- in- $\operatorname{deg}_{R}(v)$ in the graph $G_{T}$ is $f_{i}(T, \overleftarrow{R}, T)$. 
Now let $\tilde{G}_{T}=\left(V_{T}, R_{T, 1}, \ldots, R_{T, \ell}, \overleftarrow{R}_{T, 1}, \ldots, \overleftarrow{R}_{T, \ell}\right)$ be the graph obtained by taking $\overleftarrow{R}_{i}$ as the inverse of $R_{i}$. Then for each vertex $v \in V_{T}$,

- out-deg ${ }_{R}(v)=\operatorname{in-} \operatorname{deg}_{\overleftarrow{R}}(v)$ in the graph $\tilde{G}_{T}$;

- in- $\operatorname{deg}_{R}(v)=$ out-deg $\bar{K}_{\bar{R}}(v)$ in the graph $\tilde{G}_{T}$.

Similarly, by Theorem 5.2 for each $T_{i}, T_{j} \in \mathcal{T}_{\phi}$, where $j \leq i-1$, there exists a $\left(D_{T_{i} \rightarrow T_{j}}, \overleftarrow{D}_{T_{i} \rightarrow T_{j}}\right)$ biregular-complete graph

$$
G_{T_{i}, T_{j}}=\left(V_{T_{i}}, V_{T_{j}}, R_{T_{i}, T_{j}, 1}, \ldots, R_{T_{i}, T_{j}, \ell}, \overleftarrow{R}_{T_{i}, T_{j}, 1}, \ldots, \overleftarrow{R}_{T_{i}, T_{j}, \ell}\right)
$$

of size $\left(\bar{M}_{S}, \bar{M}_{T}\right)$, with $V_{T_{i}}=V_{\left(T_{i}, f_{1}\right)} \cup \cdots \cup V_{\left(T_{i}, f_{m}\right)}$ and $V_{T_{j}}=V_{\left(T_{j}, f_{1}\right)} \cup \cdots \cup V_{\left(T_{j}, f_{m}\right)}$ be the witness of the $\left(D_{T_{i} \rightarrow T_{j}}, \overleftarrow{D}_{T_{i} \rightarrow T_{j}}\right)$-biregularity. This means that for every $R \in\left\{R_{1}, \ldots, R_{\ell}, \overleftarrow{R}_{1}, \ldots, \overleftarrow{R}_{\ell}\right\}$,

- for every vertex $v \in V_{T_{i}, f}$, out-deg ${ }_{R}(v)$ in the graph $G_{T_{i}, T_{j}}$ is $f\left(T_{i}, R, T_{j}\right)$;

- for every vertex $v \in V_{T_{j}, f}$, out-deg $\operatorname{deg}_{R}(v)$ in the graph $G_{T_{i}, T_{j}}$ is $f\left(T_{i}, \overleftarrow{R}, T_{j}\right)$.

We put the orientation in every the edges in the graph $G_{T_{i}, T_{j}}$ going from $V_{T_{i}}$ to $V_{T_{j}}$. Now let $\tilde{G}_{T_{i}, T_{j}}$ be the graph obtained by adding $(u, v)$ into $\overleftarrow{R}$ in the graph $G_{T_{i}, T_{j}}$ whenever $(v, u)$ is an $R$-edge in $G_{T_{i}, T_{j}}$.

Hence, we have for each vertex $v \in V_{T_{i}} \cup V_{T_{j}}$, for each $R \in \mathcal{R}$

- out-deg ${ }_{R}(v)=\operatorname{in-} \operatorname{deg}_{\overleftarrow{R}}(v)$ in the graph $\tilde{G}_{T_{i}, T_{j}}$;

- in- $\operatorname{deg}_{R}(v)=\operatorname{out}^{-\operatorname{deg}_{\overleftarrow{R}}}(v)$ in the graph $\tilde{G}_{T_{i}, T_{j}}$.

Let $G=\left(V, R_{1}, \ldots, R_{\ell}, \overleftarrow{R}_{1}, \ldots, \overleftarrow{R}_{\ell}\right)$ be the combination of all the graphs $\tilde{G}_{T_{i}}$ 's and $\tilde{G}_{T_{i}, T_{j}}$ 's. Formally,

$$
\begin{aligned}
V & =\bigcup_{T} V\left(\tilde{G}_{t}\right) \\
R & =\bigcup_{T} R\left(\tilde{G}_{T}\right) \cup \bigcup_{T_{i}, T_{j}} R\left(\tilde{G}_{T_{i}, T_{j}}\right) \quad \text { for each } R \in\left\{R_{1}, \ldots, R_{\ell}, \overleftarrow{R}_{1}, \ldots, \overleftarrow{R}_{\ell}\right\}
\end{aligned}
$$

Moreover, we also label each vertex $v \in V$ with a subset of $\mathcal{S}$ as follows. For each $T \in \mathcal{T}_{\phi}$, for each $v \in V_{T}$, we "declare" that $v$ is labeled with a unary predicate $P \in \mathcal{S}$ if and only if $P \in T$.

We claim that $G \models \phi$. For that it is sufficient to show that for each $T \in \mathcal{T}_{\phi}$, for each $v \in V_{T}$, type $_{G}(v)=T$. The proof is divided into three cases.

- For each unary predicate $P \in \mathcal{P}$, it is by our labelling of the vertices of $G$ that $P(v)$ holds in $G$ if and only if $P \in T$.

- For each $\diamond_{R}^{l} \mu \in T$, we have

$$
\sum_{T^{\prime} \text { s.t. } T^{\prime} \ni \mu} f\left(T, R, T^{\prime}\right) \geq l
$$

number of outgoing $R$-edges from $v$. Since every function $f \in \mathcal{F}$ is consistent, $\diamond_{R}^{l} \mu \in \operatorname{type}_{G}(v)$.

- Similary, for each $\diamond_{R}^{l} \mu \notin T$, and hence $\neg\left(\diamond_{R}^{l} \mu\right) \in T$, we have

$$
\sum_{T^{\prime} \text { s.t. } T^{\prime} \ni \mu} f\left(T, R, T^{\prime}\right) \leq l-1
$$

number of outgoing $R$-edges from $v$. Since every function $f \in \mathcal{F}$ is consistent, $\diamond_{R}^{l} \mu \notin \operatorname{type}_{G}(v)$. 
Therefore the graph $G \models \phi$, and hence $N \in \operatorname{SPEC}(\phi)$.

Now we prove the direction $\operatorname{PREB}_{\phi} \supseteq \operatorname{spec}(\phi)$. Suppose $\mathfrak{A} \models \phi$ and $\mathfrak{A}$ is of size $N$. Let $\bar{M}=\left(M_{\left(T_{1}, f_{1}\right)}, \ldots, M_{\left(T_{n}, f_{m},\right)}\right)$ where $M_{(T, f)}$ be the number of elements of type $T$ from which there exist $f(T, R, S)$ number of outgoing $R$-edges towards the elements of type $S$. Take each $M_{(T, f)}$ to be the witness for $X_{(T, f)}$ for each $T \in \mathcal{T}_{\phi}$ and $f \in \mathcal{F}$. It immediately follows from Theorems 5.2 and 5.4 that $\operatorname{CON}(N, M)$ holds. Moreover, PREB-Atom $\phi(\bar{M})$ holds, since $\mathfrak{A} \models \phi$. This completes the proof of $\mathrm{PREB}_{\phi}=\operatorname{spec}(\phi)$, when $\phi$ is a basic QMLC sentence.

When $\phi \in$ QMLC is the negation of a basic QMLC sentence, say $\neg \exists^{k} \varphi$, the formula PREB-Atom $\phi$ is

$$
\sum_{(T, f) \text { s.t. } \varphi \in T} X_{(T, f)} \leq k-1
$$

which is the negation of

$$
\sum_{(T, f) \text { s.t. } \varphi \in T} X_{(T, f)} \geq k
$$

Then $\mathrm{PREB}_{\phi}=\operatorname{spec}(\phi)$ follows immediately from above.

The correctness for the case when $\phi$ is $\phi_{1} \wedge \phi_{2}$ or $\phi_{1} \vee \phi_{2}$, can be established via straightforward inductive argument. This completes our proof that $\mathrm{PREB}_{\phi}=\operatorname{spec}(\phi)$, and hence, Theorem 2.1.

\section{Proof of Theorem 5.2}

The proof of Theorem 5.2 is rather long. As a warm-up, we prove the following easy Proposition 7.1 first.

Proposition 7.1 Let $c, d \geq 0$. For every $M, N \in \mathbb{N}$, the following holds.

(a) There exists a $(c, d)$-biregular graph of size $(M, N)$ if and only if $N \geq c, M \geq d$ and $M \cdot c=N \cdot d$.

(b) There exists a $(c,-d)$-biregular graph of size $(M, N)$ if and only if $M \geq d, N \geq c$ and $M c \geq N d$.

(c) There exists a $\left(\boldsymbol{\vee}_{c}\right)$-biregular graph of size $(M, N)$ if and only if $M \geq d, N \geq c$.

Proof. Let $c, d \geq 0$, and let $M, N \in \mathbb{N}$. We first prove part (a). The "only if" direction follows from the fact that in $(c, d)$-biregular graph the number of edges is precisely $M c=N d$. That $M \geq d$ and $N \geq c$ is straightforward.

The "if" direction is as follows. Let $K=M c=N d$. Suppose also $M \geq d$ and $N \geq c$.

First, we construct the following graph.

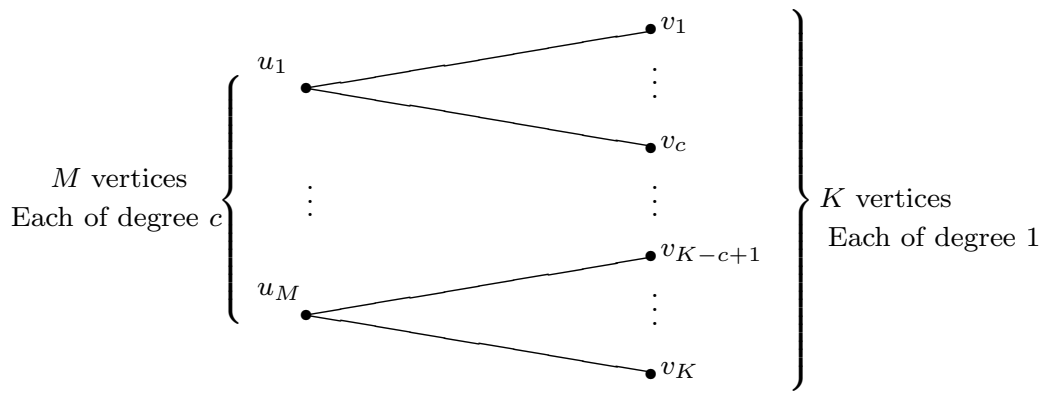

On the left side, we have $M$ vertices, and each has degree $c$. On the right side, we have $K=N d$ vertices, and each has degree 1 . We are going to merge every $d$ vertices on the right side into one vertex of degree $d$. The merging is as follows. We merge every $d$ vertices $v_{i}, v_{i+N}, \ldots, v_{i+(d-1) N}$ into one node for every $i=1, \ldots, N$. Since $K=N d$, it is possible to do such merging. Moreover, 
$N \geq c$, hence we do not have multiple edges between two vertices. Thus, we obtain the desired $(c, d)$-biregular graph of size $(M, N) \amalg$

Now we consider part (b). The "only if" direction follows from the fact that in $(c,-d)$-biregular graph the number of edges is precisely $M c$, which should be greater than $N d$. That $M \geq d$ and $N \geq c$ is straightforward.

For the "if" direction, the proof is almost the same as above. Suppose $M \geq d, N \geq c$ and $M c \geq N d$. Let $K=M c$. We first construct the bipartite graph, in which on the left side, we have $M$ vertices, each of which has degree $c$; and on the right side we have $K=M c$ vertices, each of which has degree 1.

For every $i \in\{1, \ldots, N\}$, we set the set $I_{i} \subseteq\{1, \ldots, K\}$ as follows.

$$
I_{i}:=\{i+k N \mid 1 \leq i+k N \leq K\} .
$$

Since $K=M c \geq N d$, the cardinality $\left|I_{i}\right| \geq d$ for every $1 \leq i \leq N$. Now for every $1 \leq i \leq N$, we merge the vertices $\left\{v_{j} \mid j \in I_{i}\right\}$ into one vertex. Hence, we obtain the desired $(c, \nabla)$-biregular graph of size $(M, N)$.

Now we prove part (c). The "only if" direction is straightforward. The "if" direction is as follows. Suppose $M \geq d$ and $N \geq c$. There are two cases: either $M c \geq N d$, or $M c<N d$. In the former case, we construct a $\left(c, \bullet^{-}\right)$-biregular graph of size $(M, N)$, while in the latter case, we construct a $\left(\bullet_{c}, d\right)$-biregular graph of size $(M, N)$. In either case, we obtain a $\left(\bullet_{c}, \bullet_{d}\right)$-biregular graph of size $(M, N)$. This completes our proof of Proposition 7.1

The proof of Theorem 5.2 is a generalisation of Proposition 7.1 above. It is divided into five successive steps presented in Subsections 7.1- 7.6.

- Subsection 7.1

It contains the generalisation of Proposition 7.1 to the case of $(\bar{c}, \bar{d})$-biregular, where $\bar{c}$ and $\bar{d}$ are vectors over $\mathbb{N}$. (That is, we consider the 1-type biregular graphs.)

- Subsection 7.2

In this subsection we will use the Presburger characterisation for $(\bar{c}, \bar{d})$-biregular graphs to obtain the same characterisation for $\ell$-type $(C, D)$-biregular graphs, where $C, D$ are matrices over $\mathbb{N}$.

- Subsection 7.3

In this subsection we obtain the characterisation for $(C, D)$-biregular graphs when $C, D$ contain elements from $\bullet \mathbb{N}$ assuming that the number of vertices whose degrees specified with $\bullet d$ is "big enough." It is obtained by using the characterisation in the previous Subsection 7.2 .

- Subsection 7.4

This subsection is the generalisation of Subsection 7.3, where the graphs may contain a "small" number of vertices whose degree is specified with $\boldsymbol{d}$. The idea is to encode directly those vertices in the Presburger formula. This is presented formally by our notion of partial graphs.

- Subsection 7.5 .

In this subsection we present the construction of the formula required in Theorem 5.1. It is built from the formula presented in the Subsection 7.4

- Subsection 7.6

Finally in this subsection we present the construction of the formula required in Theorem 5.2 , which is built from the from the formula in Subsection 7.5

In the following we write $\overline{1}$ to denote the vector $(1, \ldots, 1) \in \mathbb{N}^{m}$, for an appropriate $m \geq 1$. That is, $\overline{1}$ is a vector whose components are all one. For two vectors $\bar{c}=\left(c_{1}, \ldots, c_{m}\right) \in \mathbb{N}^{m}$ and $\bar{d}=\left(d_{1}, \ldots, d_{m}\right) \in \mathbb{N}^{m}$, the dot product between $\bar{c}$ and $\bar{d}$ is $\bar{c} \cdot \bar{d}=c_{1} d_{1}+\cdots+c_{m} d_{m}$.

\footnotetext{
"Note that since $M c=N d, N \geq c$ already implies $M \geq d$. That is why it is not necessary to use the fact that $M \geq d$. Of course, by symmetry, we can first build a bipartite graph in which the left side has $N$ vertices of degree $d$ and the right side has $K=M c$ vertices of degree 1 . Then to build the desired $(c, d)$-biregular graph, we make use of the fact $M \geq d$.
} 


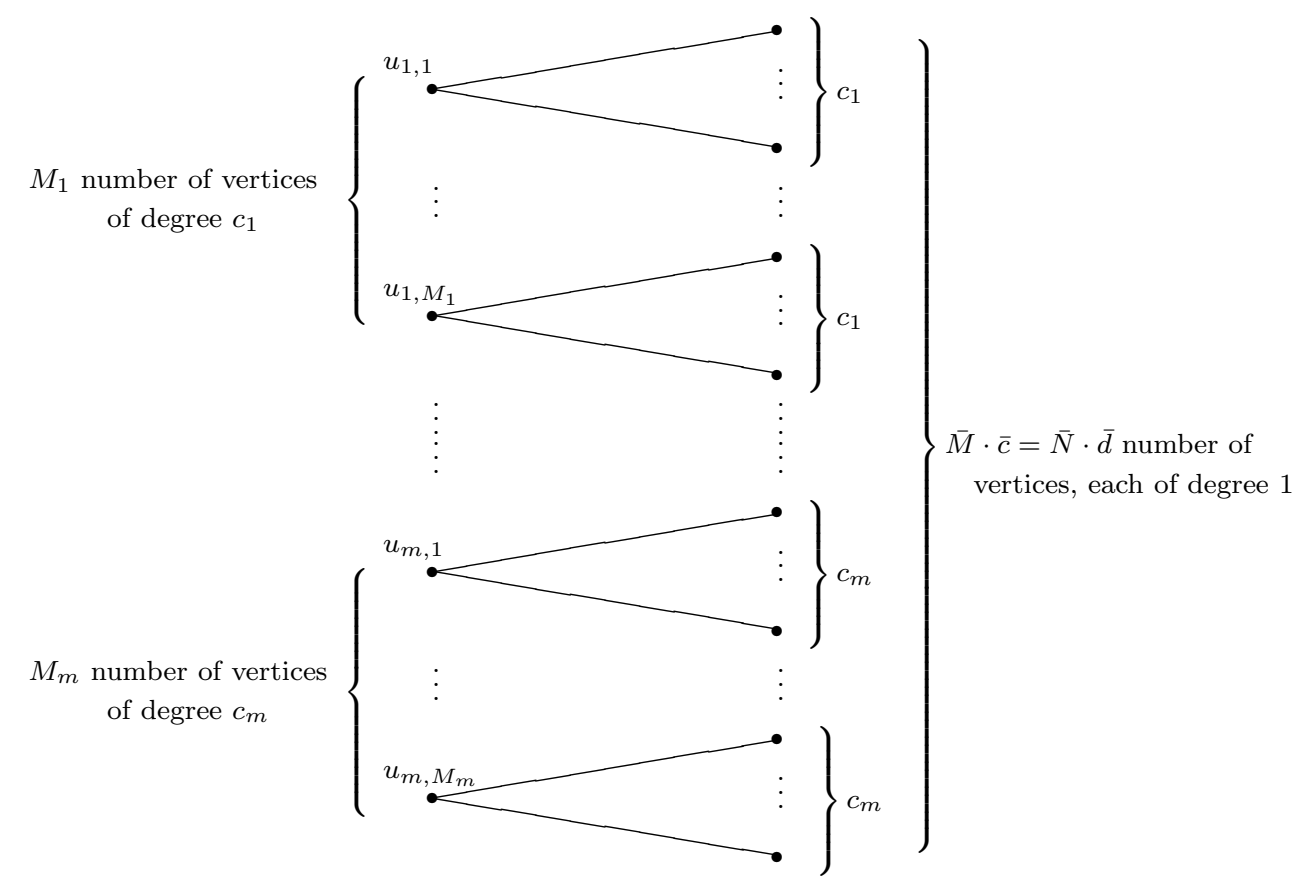

Figure 2: The preliminary graph constructed in the proof of Lemma 7.2

\subsection{When $C=\bar{c} \in \mathbb{N}^{1 \times m}$ and $D=\bar{d} \in \mathbb{N}^{1 \times n}$}

In this subsection we consider the case when $C$ and $D$ consist of only one vector each. In this case, we are going to write $(\bar{c}, \bar{d})$-biregular graph, where $\bar{c}$ and $\bar{d}$ are the only vectors of $C$ and $D$, respectively.

Lemma 7.2 Let $\bar{c} \in \mathbb{N}^{m}$ and $\bar{d} \in \mathbb{N}^{n}$ and both do not contain zero entry. For each $\bar{M} \in \mathbb{N}^{m}$ and $\bar{N} \in \mathbb{N}^{n}$ such that $\bar{M} \cdot \overline{1}+\bar{N} \cdot \overline{1} \geq 2(\bar{c} \cdot \overline{1})(\bar{d} \cdot \overline{1})+3$, the following holds. There exists a $(\bar{c}, \bar{d})$-biregular graph of size $(\bar{M}, \bar{N})$ if and only if $\bar{M} \cdot \bar{c}=\bar{N} \cdot \bar{d}$.

Proof. Let $\bar{c} \in \mathbb{N}^{m}$ and $\bar{d} \in \mathbb{N}^{n}$ and both do not contain zero entry. Let $\bar{M} \in \mathbb{N}^{m}, \bar{N} \in \mathbb{N}^{n}$ such that $\bar{M} \cdot \overline{1}+\bar{N} \cdot \overline{1} \geq 2(\bar{c} \cdot \overline{1})(\bar{d} \cdot \overline{1})+3$.

The "only if" direction is straightforward. If $G$ is a $(\bar{c}, \bar{d})$-biregular graph of size $(\bar{M}, \bar{N})$, then the number of edges in $G$ is precisely $\bar{M} \cdot \bar{c}=\bar{N} \cdot \bar{d}$.

Now we prove the "if" part. Suppose $\bar{M} \in \mathbb{N}^{m}, \bar{N} \in \mathbb{N}^{n}$ such that $\bar{M} \cdot \bar{c}=\bar{N} \cdot \bar{d}$. Let $\bar{c}=\left(c_{1}, \ldots, c_{m}\right)$ and $\bar{d}=\left(d_{1}, \ldots, d_{n}\right)$, and $\bar{M}=\left(M_{1}, \ldots, M_{m}\right)$ and $\bar{N}=\left(N_{1}, \ldots, N_{n}\right)$.

We are going to construct a $(\bar{c}, \bar{d})$-biregular graph of size $(\bar{M}, \bar{N})$. We first construct a preliminary bipartite graph $G$ pictured in Figure 2, That is, the left side has $\bar{M} \cdot \overline{1}$ vertices, and there are $M_{1}$ vertices of degree $c_{1}, M_{2}$ nodes of degree $c_{2}$, etc. The right side has $\bar{M} \cdot \bar{c}$ number of vertices, each of degree one.

We are going to do some merging of the vertices on the right side so that there are exactly $N_{1}$ vertices of degree $d_{1}, N_{2}$ vertices of degree $d_{2}$, etc. We do the following. We "group" the vertices on the right side into $V_{1}, \ldots, V_{n}$ where $V_{1}$ has $N_{1} d_{1}$ vertices, $V_{2}$ has $N_{2} d_{2}$ vertices, etc. Such grouping is possible because $\bar{M} \cdot \bar{c}=\bar{N} \cdot \bar{d}$.

For each $i \in\{1, \ldots, n\}$, we do the following. We merge $d_{i}$ vertices in $V_{i}$ into one vertex, so that each vertex in $V_{i}$ has degree $d_{i}$. Let $V_{i}=\left\{v_{i, 1}, \ldots, v_{i, K_{i}}\right\}$ where $K_{i}=N_{i} d_{i}$. We merge the vertices $v_{1}, v_{N_{i}+1}, v_{2 N_{i}+1}, \ldots, v_{\left(d_{i}-1\right) N_{i}+1}$ into one vertex; the vertices $v_{2}, v_{N_{i}+2}, v_{2 N_{i}+2}, \ldots, v_{\left(d_{i}-1\right) N_{i}+2}$ into one vertex; and so on. 
After such merging, each vertex in $V_{i}$ has degree $d_{i}$. However, it is possible that after we do the merging, we have "parallel" edges, i.e. more than one edges between two vertices. (See the left side of the illustration below.) We are going to "remove" such parallel edges one by one until there are no more parallel edges.

Suppose we have parallel edges between the vertices $u$ and $v$. We pick an edge $\left(u^{\prime}, v^{\prime}\right)$ such that $u^{\prime}$ is not adjacent to $v$ and $v^{\prime}$ is not adjacent to $u$. (See the left side of the illustration below.)
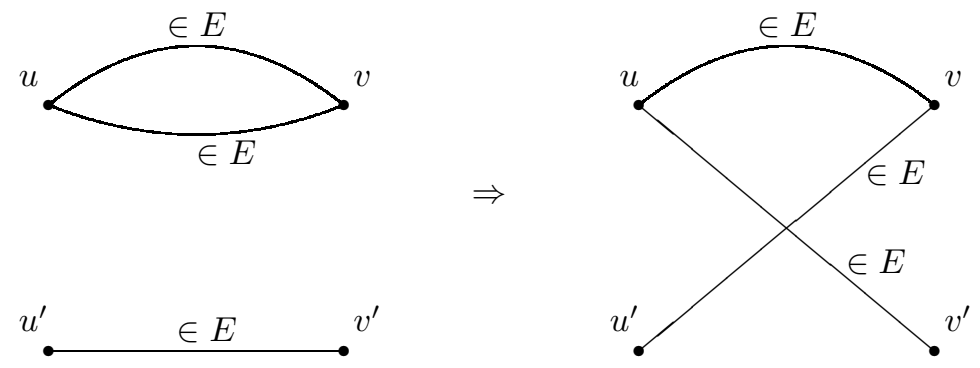

Such an edge $\left(u^{\prime}, v^{\prime}\right)$ exists since the number of vertices reachable in distance 2 from the vertices $u$ and $v$ is $\leq 2(\bar{c} \cdot \overline{1})(\bar{d} \cdot \overline{1})+2$ and the number of vertices is $\bar{M} \cdot \overline{1}+\bar{N} \cdot \overline{1} \geq 2(\bar{c} \cdot \overline{1})(\bar{d} \cdot \overline{1})+3$ and the fact that none of the vertices are of zero degree. (Here we make use of the fact that neither $\bar{c}$ nor $\bar{d}$ contain zero entry.)

Now we delete the edges $\left(u^{\prime}, v^{\prime}\right)$ and one of the parallel edge $(u, v)$, replace it with the edges $\left(u, v^{\prime}\right)$ and $\left(u^{\prime}, v\right)$, as illustrated on the right side of the illustration above. We perform such operation until there are no more parallel edges. This completes the proof of Lemma 7.2 .

The following theorem is a straightforward application of Lemma 7.2

Theorem 7.3 For every $\bar{c} \in \mathbb{N}^{m}$ and $\bar{d} \in \mathbb{N}^{n}$, there exists a Presburger formula $\operatorname{BiRE}_{(\bar{c}, \bar{d})}(\bar{X}, \bar{Y})$, where $\bar{X}=\left(X_{1}, \ldots, X_{m}\right)$ and $\bar{Y}=\left(Y_{1}, \ldots, Y_{n}\right)$ such that the following holds. There exists a $(\bar{c}, \bar{d})$-biregular graph of size $(\bar{M}, \bar{N})$ if and only if the sentence $\operatorname{BiREG}_{(\bar{c}, \bar{d})}(\bar{M}, \bar{N})$ holds.

Proof. The proof is a direct application of Lemma 7.2. We assume that all the entries in $\bar{c}$ and $\bar{d}$ are not zero. Otherwise, we do the following. Suppose $\bar{c}=\left(c_{1}, \ldots, c_{m}\right), \bar{d}=\left(d_{1}, \ldots, d_{n}\right)$ and let $I=\left\{i \mid c_{i}=0\right\}$ and $J=\left\{j \mid d_{j}=0\right\}$. We define

$$
\operatorname{BiREG}_{\bar{c}, \bar{d}}(\bar{X}, \bar{Y}):=\bigwedge_{i \in I} X_{i} \geq 0 \wedge \bigwedge_{j \in J} Y_{j} \geq 0 \wedge \operatorname{BiREG}_{\bar{c}^{\prime}, \bar{d}^{\prime}}\left(\bar{X}^{\prime}, \bar{Y}^{\prime}\right),
$$

where $\bar{c}^{\prime}$ and $\bar{X}^{\prime}$ are the vectors $\bar{c}$ and $\bar{X}$ without the entries in $I$, respectively, and $\bar{d}^{\prime}$ and $\bar{Y}^{\prime}$ are the vectors $\bar{d}$ and $\bar{Y}$ without the entries in $J$, respectively.

For $\bar{c} \in \mathbb{N}^{m}$ and $\bar{d} \in \mathbb{N}^{n}$ which do not contain zero entry, we define the following set $H$.

$$
H:=\left\{\begin{array}{l|l}
(\bar{M}, \bar{N}) & \begin{array}{l}
\bar{M} \cdot \overline{1}+\bar{N} \cdot \overline{1} \leq 2(\bar{c} \cdot \overline{1})(\bar{d} \cdot \overline{1})+2 \text { and } \\
\text { there exists a }(\bar{c}, \bar{d}) \text {-biregular graph of size }(\bar{M}, \bar{N})
\end{array}
\end{array}\right\}
$$

Such set can be computed greedily since the number of $(\bar{M}, \bar{N})$ such that $\bar{M} \cdot \overline{1}+\bar{N} \cdot \overline{1} \leq 2(\bar{c} \cdot \overline{1})(\bar{d} \cdot \overline{1})+2$ is bounded.

Now we define the formula $\operatorname{BiREG}_{\bar{c}, \bar{d}}(\bar{X}, \bar{Y})$ as follows.

$$
(\bar{M} \cdot \overline{1}+\bar{N} \cdot \overline{1} \geq 2(\bar{c} \cdot \overline{1})(\bar{d} \cdot \overline{1})+3 \wedge(\bar{X} \cdot \bar{c}=\bar{Y} \cdot \bar{d})) \vee \bigvee_{(\bar{M}, \bar{N}) \in H} \bar{X}=\bar{M} \wedge \bar{Y}=\bar{N}
$$

The formula is a Presburger formula since $\bar{c}$ and $\bar{d}$ are constants. Since $\bar{c}, \bar{d}$ do not contain zero entry, we can apply Lemma 7.2 to obtain the correctness of $\operatorname{BiREG}_{\bar{c}, \bar{d}}(\bar{X}, \bar{Y})$. This completes our proof of Theorem 7.3 . 


\subsection{When $C \in \mathbb{N}^{\ell \times m}$ and $D \in \mathbb{N}^{\ell \times n}$}

Theorem 7.4 below is the generalisation of Theorem 7.3 to the case where $\ell \geq 1$. Recall that for a matrix $C \in \mathbb{N}^{\ell \times m}$, we write $C \cdot \overline{1}$ to denote the sum of all the entries in $C$.

Theorem 7.4 For every $C \in \mathbb{N}^{\ell \times m}$ and $D \in \mathbb{N}^{\ell \times n}$, there exists a Presburger formula $\operatorname{BiREG} C, D(\bar{X}, \bar{Y})$, where $\bar{X}=\left(X_{1}, \ldots, X_{m}\right)$ and $\bar{Y}=\left(Y_{1}, \ldots, Y_{n}\right)$ such that the following holds. There exists a $(C, D)$ biregular $\ell$-type graph of size $(\bar{M}, \bar{N})$ if and only if the sentence $\operatorname{BiRE}_{C, D}(\bar{M}, \bar{N})$ holds.

Proof. Let $C \in \mathbb{N}^{\ell \times m}$ and $D \in \mathbb{N}^{\ell \times n}$ be the given matrices. For simplicity, we assume that both $C$ and $D$ do not contain zero column. If column $i$ in matrix $C$ (or, $D$, respectively) is zero column, then we add the constraint $X_{i} \geq 0$ (or, $Y_{i} \geq 0$, respectively) and ignore that column.

Let $\bar{c}_{1}, \ldots, \bar{c}_{\ell}$ and $\bar{d}_{1}, \ldots, \bar{d}_{\ell}$ be the row vectors of $C$ and $D$, respectively. For a vector $\bar{t}=$ $\left(t_{1}, \ldots, t_{m}\right) \in \mathbb{N}^{m}$, we define the characteristic vector of $\bar{t}$ as $\chi(\bar{t}):=\left(b_{1}, \ldots, b_{m}\right) \in\{0,1\}^{m}$ where $b_{i}=0$ if $t_{i}=0$, and $b_{i}=1$ if $t_{i} \neq 0$.

We first define the following set.

$$
H_{C, D}:=\left\{(\bar{M}, \bar{N}) \mid \begin{array}{l}
\bar{M} \cdot \overline{1}+\bar{N} \cdot \overline{1}<2 \ell(C \cdot \overline{1})(D \cdot \overline{1})+3 \ell \text { and } \\
\text { there exists a }(C, D) \text {-biregular graph of size }(\bar{M}, \bar{N})
\end{array}\right\}
$$

Again, such set can be computed greedily since the number of $(\bar{M}, \bar{N})$ such that $\bar{M} \cdot \overline{1}+\bar{N} \cdot \overline{1}<$ $2 \ell(C \cdot \overline{1})(D \cdot \overline{1})+3 \ell$ is bounded.

Then, the formula $\operatorname{BiREG}_{C, D}(\bar{X}, \bar{Y})$ can be defined inductively as follows. When $\ell=1$,

$$
\operatorname{BiREG}_{C, D}(\bar{X}, \bar{Y}):=\operatorname{BiREG}_{\bar{c}_{1}, \bar{d}_{1}}(\bar{X}, \bar{Y})
$$

When $\ell \geq 2$,

$$
\begin{aligned}
& \operatorname{BiREG}_{C, D}(\bar{X}, \bar{Y}):=\bigvee_{(\bar{M}, \bar{N}) \in H_{C, D}} \bar{X}=\bar{M} \wedge \bar{Y}=\bar{N} \\
& \vee \bigvee_{1 \leq j \leq \ell}\left(\bar{X} \cdot \chi\left(\bar{c}_{j}\right)+\bar{Y} \cdot \chi\left(\bar{d}_{j}\right) \geq 2(C \cdot \overline{1})(D \cdot \overline{1})+3\right. \\
& \wedge \operatorname{BiREG}_{C-\bar{c}_{j}, D-\bar{d}_{j}}(\bar{X}, \bar{Y}) \\
& \left.\wedge \operatorname{BiREG}_{\bar{c}_{j}, \bar{d}_{j}}(\bar{X}, \bar{Y})\right)
\end{aligned}
$$

where $C-\bar{c}_{j}, D-\bar{d}_{j}$ denote the matrices $C$ and $D$ without row $j$, respectively.

We are going to prove that there exists a $(C, D)$-biregular graph of size $(\bar{M}, \bar{N})$ if and only if the statement $\operatorname{BiREG}_{C, D}(\bar{M}, \bar{N})$ holds. The proof is by induction on $\ell$. The basis $\ell=1$ has been established in Theorem 7.3 . For the induction step, we assume that it holds for the case of $\ell-1$ and we are going to prove the case $\ell$.

We first prove the "only if" direction. Suppose $G=\left(U, V, E_{1}, \ldots, E_{\ell}\right)$ is $(C, D)$-biregular of size $(\bar{M}, \bar{N})$. If $(\bar{M}, \bar{N}) \in H_{C, D}$, then $\operatorname{BiREG}_{C, D}(\bar{M}, \bar{N})$ holds. So suppose $(\bar{M}, \bar{N}) \notin I_{C, D}$ and $\bar{M} \cdot \overline{1}+\bar{N} \cdot \overline{1} \geq 2 \ell(C \cdot \overline{1})(D \cdot \overline{1})+3 \ell$. Since $C$ and $D$ do not contain zero column, there exists $j \in\{1, \ldots, \ell\}$ such that $\bar{M} \cdot \chi\left(\bar{c}_{j}\right)+\bar{N} \cdot \chi\left(\bar{d}_{j}\right) \geq 2(C \cdot \overline{1})(D \cdot \overline{1})+3$. Moreover, if $G=\left(U, V, E_{1}, \ldots, E_{\ell}\right)$ is $(C, D)$-biregular of size $(\bar{M}, \bar{N})$, then $G$ is also $\left(C-\bar{c}_{j}, D-\bar{d}_{j}\right)$-biregular and $\left(\bar{c}_{j}, \bar{d}_{j}\right)$-biregular. By the induction hypothesis, both $\operatorname{BiREG}_{C-\bar{c}_{j}, D-\bar{d}_{j}}(\bar{M}, \bar{N})$ and $\operatorname{BiREG}_{\bar{c}_{j}, \bar{d}_{j}}(\bar{M}, \bar{N})$ hold.

We now prove the "if" direction. Suppose $\operatorname{BiREG}_{C, D}(\bar{M}, \bar{N})$ holds. If $(\bar{M}, \bar{N}) \in H_{C, D}$, then there exists a $(C, D)$-biregular graph of size $(\bar{M}, \bar{N})$ and we are done. So suppose $(\bar{M}, \bar{N}) \notin H_{C, D}$. Hence there exists $j \in\{1, \ldots, \ell\}$ such that

$$
\begin{aligned}
& \bar{M} \cdot \chi\left(\bar{c}_{j}\right)+\bar{N} \cdot \chi\left(\bar{d}_{j}\right) \geq 2(C \cdot \overline{1})(D \cdot \overline{1})+3 \\
& \wedge \operatorname{BiREG}_{C-\bar{c}_{j}, D-\bar{d}_{j}}(\bar{M}, \bar{N}) \wedge \operatorname{BiREG}_{\bar{c}_{j}, \bar{d}_{j}}(\bar{M}, \bar{N})
\end{aligned}
$$

For simplicity, we assume that $j=\ell$. By the induction hypothesis, there exists a $\left(C-\bar{c}_{\ell}, D-\bar{d}_{\ell}\right)$ biregular graph $G_{1}=\left(U_{1}, V_{1}, E_{1}, \ldots, E_{\ell-1}\right)$ of size $(\bar{M}, \bar{N})$, and by definition, $E_{1}, \ldots, E_{\ell-1}$ are 
pairwise disjoint. By Theorem 7.3, there exists a $\left(\bar{c}_{\ell}, \bar{d}_{\ell}\right)$-biregular graph $G_{2}=\left(U_{2}, V_{2}, E_{\ell}\right)$ of size $(\bar{M}, \bar{N})$. We can assume that $U_{1}=U_{2}=U$ and $V_{1}=V_{2}=V$ since $G_{1}$ and $G_{2}$ are of the same size $(\bar{M}, \bar{N})$.

We are going to combine $G_{1}$ and $G_{2}$ into one graph to get an $\ell$-type $(C, D)$-biregular graph $G=$ $\left(U, V, E_{1}, \ldots, E_{\ell}\right)$ of size $(\bar{M}, \bar{N})$. If $E_{\ell} \cap\left(E_{1} \cup \cdots \cup E_{\ell-1}\right)=\emptyset$, then the graph $G=\left(U, V, E_{1}, \ldots, E_{\ell}\right)$ is the desired $(C, D)$-biregular $\ell$-type graph of size $(\bar{M}, \bar{N})$, and we are done.

Now suppose $E_{\ell} \cap\left(E_{1} \cup \cdots \cup E_{\ell-1}\right) \neq \emptyset$. We are going to construct another graph $G_{2}^{\prime}=\left(U, V, E_{\ell}^{\prime}\right)$ such that

$$
\left|E_{\ell}^{\prime} \cap\left(E_{1} \cup \cdots \cup E_{\ell-1}\right)\right|<\left|E_{\ell} \cap\left(E_{1} \cup \cdots \cup E_{\ell-1}\right)\right|
$$

We do this repeatedly until at the end we obtain a graph $G_{2}^{\prime \prime}=\left(V, E_{\ell}^{\prime \prime}\right)$ such that $E_{\ell}^{\prime \prime} \cap\left(E_{1} \cup \cdots \cup\right.$ $\left.E_{\ell-1}\right)=\emptyset$.

Let $(u, v) \in E_{\ell} \cap\left(E_{1} \cup \cdots \cup E_{\ell-1}\right)$. The number of vertices reachable in from $u$ and $v$ within distance 2 (by any of edges in $\left.E_{1}, \ldots, E_{\ell}\right)$ is $\leq 2(C \cdot \overline{1})(D \cdot \overline{1})+2$. Since $\bar{M} \cdot \chi\left(\bar{c}_{\ell}\right)+\bar{N} \cdot \chi\left(\bar{d}_{\ell}\right) \geq$ $2(C \cdot \overline{1})(D \cdot \overline{1})+3$, there exists $\left(u^{\prime}, v^{\prime}\right) \in E_{\ell}$ such that $\left(u, u^{\prime}\right),\left(v, v^{\prime}\right) \notin E_{1} \cup \cdots \cup E_{\ell}$. See the left side of the illustration below.

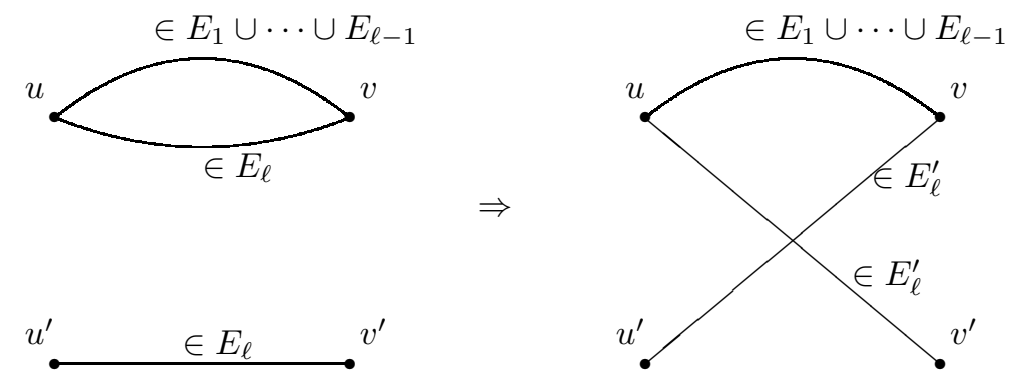

Now we define $E_{\ell}^{\prime}$ by deleting the edges $(u, v),\left(u^{\prime}, v^{\prime}\right)$ from $E_{\ell}$, while adding the edges $\left(u, v^{\prime}\right),\left(u^{\prime}, v\right)$ into $E_{\ell}$. Formally,

$$
E_{\ell}^{\prime}:=\left(E_{\ell}-\left\{(u, v),\left(u^{\prime}, v^{\prime}\right)\right\}\right) \cup\left\{\left(u, u^{\prime}\right),\left(v, v^{\prime}\right)\right\}
$$

See the right side of the illustration above.

Now it is straightforward that $G^{\prime}=\left(U, V, E_{\ell}^{\prime}\right)$ is still a $\bar{d}_{\ell}$-regular graph of size $\bar{N}$, while

$$
\left|E_{\ell}^{\prime} \cap\left(E_{1} \cup \cdots \cup E_{\ell-1}\right)\right|<\left|E_{\ell} \cap\left(E_{1} \cup \cdots \cup E_{\ell-1}\right)\right|
$$

We perform this operation until $E_{\ell+1} \cap\left(E_{1} \cup \cdots \cup E_{\ell}\right)=\emptyset$. This completes the proof of Theorem 7.4.

\subsection{For $C \in \mathbb{B}^{\ell \times n}$ and $D \in \mathbb{B}^{\ell \times m}$ when the number of vertices is "big enough"}

Let $C \in \mathbb{B}^{\ell \times m}$ and $D \in \mathbb{B}^{\ell \times n}$, where $\bar{c}_{1}, \ldots, \bar{c}_{\ell}$ and $\bar{d}_{1}, \ldots, \bar{d}_{\ell}$ are the row vectors of $C$ and $D$, respectively.

Two vectors $\bar{M}=\left(M_{1}, \ldots, M_{m}\right) \in \mathbb{N}^{m}$ and $\bar{N}=\left(N_{1}, \ldots, N_{n}\right) \in \mathbb{N}^{n}$ are "big enough" with respect to $C, D$, if the following inequalities hold.

$$
\begin{aligned}
\wedge & \bigwedge_{1 \leq i \leq \ell}\left(\bar{M} \cdot \chi\left(\bar{c}_{i}\right)+\bar{N} \cdot \chi\left(\bar{d}_{i}\right) \geq 2(C \cdot \overline{1})(D \cdot \overline{1})+3\right) \\
\wedge & \bigwedge_{1 \leq i \leq \ell}\left(\left(\sum_{j \text { such that } D_{i, j} \in \mathbb{N}} N_{j}\right) \geq \max _{1 \leq j \leq m}\left(C_{i, j}\right)\right) \\
\wedge & \left.\bigwedge_{1 \leq i \leq \ell}\left(\sum_{j \text { such that } C_{i, j} \in \mathbb{N}} M_{j}\right) \geq \max _{1 \leq j \leq m}\left(D_{i, j}\right)\right)
\end{aligned}
$$


We need a few notations. In the following for a positive integer $\ell, \mathbf{I}_{\ell}$ denotes the $(\ell \times \ell)$ identity matrix. For $\downarrow d \in \mathbb{N}$, we write $\lfloor d\rfloor$ to denote the number $d$. By default, we set $\lfloor d\rfloor=d$. We also define the + operations on $\mathbb{B}$ as follows.

$$
\begin{aligned}
d_{1}+d_{2} & =\left(d_{1}+d_{2}\right) \\
\bullet d_{1}+d_{2} & =d_{1}+d_{2}=d_{1}+d_{2}={ }=\left(d_{1}+d_{2}\right)
\end{aligned}
$$

We extend $\lfloor\cdot\rfloor$ and + to vectors and matrices over $\mathbb{B}$ in the natural way, where they are applied componentwise. For two vectors $\bar{t}_{1}, \bar{t}_{2} \in \mathbb{B}^{m}$, we define the $\operatorname{dot}$ product $\bar{t}_{1} \cdot \bar{t}_{2}$ as $\left\lfloor\bar{t}_{1}\right\rfloor \cdot\left\lfloor\bar{t}_{2}\right\rfloor$. For a matrix $D \in \mathbb{B}^{\ell \times m}$, we write $D \cdot \overline{1}$ to denote the sum $\sum_{i, j}\left\lfloor D_{i, j}\right\rfloor$.

The lemma below characterises the existence of $(C, D)$-biregular graph of size $(\bar{M}, \bar{N})$, where $\bar{M}, \bar{N}$ are big enough with respect to $(C, D)$ and that for every row $i$, either the row- $i$ of $C$ or of $D$ contains only elements from $\mathbb{N}$.

Lemma 7.5 Let $C \in\left(\begin{array}{l}C^{(1)} \\ C^{(2)} \\ C^{(3)}\end{array}\right) \in \mathbb{B}^{\ell \times m}$ and $D \in\left(\begin{array}{c}D^{(1)} \\ D^{(2)} \\ D^{(3)}\end{array}\right) \in \mathbb{B}^{\ell \times n}$ where $\ell=\ell_{1}+\ell_{2}+\ell_{3}$ and

- $C^{(1)} \in \mathbb{N}^{\ell_{1} \times m}$ and $D^{(1)} \in \mathbb{N}^{\ell_{1} \times n}$;

- $C^{(2)} \in \mathbb{N}^{\ell_{2} \times m}$ and $D^{(2)} \in \mathbb{B}^{\ell_{2} \times n}$ and every row in $D^{(2)}$ contains an element of $\mathbb{N}$;

- $C^{(3)} \in \mathbb{B}^{\ell_{3} \times m}$ and $D^{(3)} \in \mathbb{N}^{\ell_{3} \times n}$ and every row in $C^{(3)}$ contains an element of $\mathbb{N}$.

Let $\bar{M} \in \mathbb{N}^{m}$ and $\bar{N} \in \mathbb{N}^{n}$ be big enough with respect to $C$ and $D$. Then the following holds. There is $a(C, D)$-biregular graph of size $(\bar{M}, \bar{N})$ if and only if $\operatorname{BiREG}_{C^{\prime}, D^{\prime}}\left(\left(\bar{M}, K_{1}, \ldots, K_{\ell_{3}}\right),\left(\bar{N}, L_{1}, \ldots, L_{\ell_{2}}\right)\right)$ holds, where

- $C^{\prime}=\left(\begin{array}{c|c}C^{(1)} & 0 \\ \hline C^{(2)} & 0 \\ \hline\left\lfloor C^{(3)}\right\rfloor & \mathbf{I}_{\ell_{3}}\end{array}\right) \in \mathbb{N}^{\ell \times\left(m+\ell_{3}\right)}$ and $D^{\prime}=\left(\begin{array}{c|c}D^{(1)} & 0 \\ \hline\left\lfloor D^{(2)}\right\rfloor & \mathbf{I}_{\ell_{2}} \\ \hline D^{(3)} & 0\end{array}\right) \in \mathbb{N}^{\ell \times\left(n+\ell_{2}\right)}$

- each $K_{i}=\bar{d}_{\ell_{1}+\ell_{2}+i} \cdot \bar{N}-\left\lfloor\bar{c}_{\ell_{1}+\ell_{2}+i}\right\rfloor \cdot \bar{M}$,

- each $L_{i}=\bar{c}_{\ell_{1}+i} \cdot \bar{M}-\left\lfloor\bar{d}_{\ell_{1}+i}\right\rfloor \cdot \bar{N}$.

Proof. Let $C \in\left(\begin{array}{c}C^{(1)} \\ C^{(2)} \\ C^{(3)}\end{array}\right) \in \mathbb{B}^{\ell \times m}$ and $D \in\left(\begin{array}{c}D^{(1)} \\ D^{(2)} \\ D^{(3)}\end{array}\right) \in \mathbb{B}^{\ell \times n}$ and $\ell_{1}, \ell_{2}, \ell_{3}, \bar{M} \in \mathbb{N}^{m}$ and $\bar{N} \in \mathbb{N}^{n}$ be as in the premises. We also assume that $\bar{c}_{1}, \ldots, \bar{c}_{\ell}$ and $\bar{d}_{1}, \ldots, \bar{d}_{\ell}$ are the row vectors of $C$ and $D$, respectively.

Before we present our proof, we have to remark here that we do not need the condition that $\bar{M}$ and $\bar{N}$ are big enough to establish the "only if" direction. For the "if" direction, we only need Inequalities 2 and 3. Inequality 1 is needed only to established Theorem 7.6 .

We start with the "only if" direction. Suppose $G=\left(U, V, E_{1}, \ldots, E_{\ell}\right)$ is a $(C, D)$-biregular graph of size $(\bar{M}, \bar{N})$. This means there exist a partition $U=U_{1} \cup \cdots \cup U_{m}$ and a partition $V=V_{1} \cup \cdots \cup V_{n}$ such that for each $i=1, \ldots, \ell$,

- for each $j=1, \ldots, m$, for each $u \in U_{j}, \operatorname{deg}_{E_{i}}(u)=C_{i, j}$;

- for each $j=1, \ldots, n$, for each $v \in V_{j}, \operatorname{deg}_{E_{i}}(v)=D_{i, j}$.

Now, the following holds.

- For each $i=\ell_{1}+1, \ldots, \ell_{1}+\ell_{2}$, the number of $E_{i}$-edges in $G$ is $\bar{c}_{i} \cdot \bar{M}$, which should be greater than $\left\lfloor\bar{d}_{i}\right\rfloor \cdot \bar{N}$. We set $L_{i-\ell_{1}}=\bar{c}_{i} \cdot \bar{M}-\left\lfloor\bar{d}_{i}\right\rfloor \cdot \bar{N}$.

- For each $i=\ell_{1}+\ell_{2}+1, \ldots, \ell_{1}+\ell_{2}+\ell_{3}$, the number of $E_{i}$-edges in $G$ is $\bar{d}_{i} \cdot \bar{N}$, which should be greater than $\left\lfloor\bar{c}_{i}\right\rfloor \cdot \bar{M}$. We set $K_{i-\ell_{1}-\ell_{2}}=\bar{d}_{i} \cdot \bar{N}-\left\lfloor\bar{c}_{i}\right\rfloor \cdot \bar{M}$. 
Let $C^{\prime}, D^{\prime}$ be as defined in the lemma, and $\bar{K}=\left(K_{1}, \ldots, K_{\ell_{3}}\right)$ and $\bar{L}=\left(L_{1}, \ldots, L_{\ell_{2}}\right)$. We construct a $\left(C^{\prime}, D^{\prime}\right)$-biregular graph of size $((\bar{M}, \bar{K}),(\bar{N}, \bar{L}))$ as follows.

- For each $i=\ell_{1}+1, \ldots, \ell_{1}+\ell_{2}$, for each $j=1, \ldots, n$, for each vertex $v \in V_{j}$, if $\operatorname{deg}_{E_{i}}(v)>$ $\left\lfloor D_{i, j}\right\rfloor$, then we "split" $v$ into $v_{0}, v_{1}, \ldots, v_{k}$ vertices, where

$-k=\operatorname{deg}_{E_{i}}(v)-\left\lfloor D_{i, j}\right\rfloor$,

- $\operatorname{deg}_{E_{i}}\left(v_{0}\right)=\left\lfloor D_{i, j}\right\rfloor$, and for each $i^{\prime} \neq i, \operatorname{deg}_{E_{i^{\prime}}}\left(v_{0}\right)=\operatorname{deg}_{E_{i^{\prime}}}(v)$,

$-\operatorname{deg}_{E_{i}}\left(v_{1}\right)=\operatorname{deg}_{E_{i}}\left(v_{2}\right)=\cdots=\operatorname{deg}_{E_{i}}\left(v_{k}\right)=1$, and for each $i^{\prime} \neq i, \operatorname{deg}_{E_{i^{\prime}}}\left(v_{1}\right)=$ $\operatorname{deg}_{E_{i^{\prime}}}\left(v_{2}\right)=\cdots=\operatorname{deg}_{E_{i^{\prime}}}\left(v_{k}\right)=0$.

- Similarly, for each $i=\ell_{1}+\ell_{2}+1, \ldots, \ell_{1}+\ell_{2}+\ell_{3}$, for each $j=1, \ldots, m$, for each vertex $u \in U_{j}$, if $\operatorname{deg}_{E_{i}}(u)>\left\lfloor C_{i, j}\right\rfloor$, then we "split" $u$ into $u_{0}, u_{1}, \ldots, u_{k}$ vertices, where

- $k=\operatorname{deg}_{E_{i}}(u)-\left\lfloor D_{i, j}\right\rfloor$,

$-\operatorname{deg}_{E_{i}}\left(u_{0}\right)=\left\lfloor D_{i, j}\right\rfloor$, and for each $i^{\prime} \neq i, \operatorname{deg}_{E_{i^{\prime}}}\left(u_{0}\right)=\operatorname{deg}_{E_{i^{\prime}}}(u)$,

$-\operatorname{deg}_{E_{i}}\left(u_{1}\right)=\operatorname{deg}_{E_{i}}\left(u_{2}\right)=\cdots=\operatorname{deg}_{E_{i}}\left(u_{k}\right)=1$, and for each $i^{\prime} \neq i, \operatorname{deg}_{E_{i^{\prime}}}\left(u_{1}\right)=$ $\operatorname{deg}_{E_{i^{\prime}}}\left(u_{2}\right)=\cdots=\operatorname{deg}_{E_{i^{\prime}}}\left(u_{k}\right)=0$.

It should be obvious that the resulting graph is a $\left(C^{\prime}, D^{\prime}\right)$-biregular graph of size $((\bar{M}, \bar{K}),(\bar{N}, \bar{L}))$.

Now we prove the "if" direction. Suppose $\operatorname{BiREG}_{C^{\prime}, D^{\prime}}((\bar{M}, \bar{K}),(\bar{N}, \bar{L}))$ holds, where each $L_{i}=$ $\bar{c}_{i} \cdot \bar{M}-\left\lfloor\bar{d}_{i}\right\rfloor \cdot \bar{N}$ and $K_{i}=\bar{d}_{i} \cdot \bar{N}-\left\lfloor\bar{c}_{i}\right\rfloor \cdot \bar{M}$.

By Theorem 7.4 there exists a $\left(C^{\prime}, D^{\prime}\right)$-biregular graph $G$ of size $((\bar{M}, \bar{K}),(\bar{N}, \bar{L}))$. Let $G=$ $\left(U \cup A, V \cup B, E_{1}, \ldots, E_{\ell}\right)$, where $U \cup A=U_{1} \cup \cdots \cup U_{m} \cup A_{1} \cup \cdots \cup A_{\ell_{3}}$ and $V \cup B=V_{1} \cdots \cdots \cup$ $V_{n} \cup B_{1} \cup \cdots \cup B_{\ell_{2}}$ are the witness of the $\left(C^{\prime}, D^{\prime}\right)$-biregularity.

To construct a $(C, D)$-biregular graph of size $(\bar{M}, \bar{N})$, we do the following. For each vertex $u \in U$ adjacent by $E_{i}$-edges to, say, $s$ vertices in $B$, we pick $s$ vertices $v_{1}, \ldots, v_{s}$ from the set

$$
\bigcup_{j \text { such that } d_{i, j} \in \mathbb{N}} V_{j}
$$

Such $s$ vertices exist since by Inequality 2., $\sum_{j \text { such that } d_{i, j} \in \mathbb{N}} N_{j}$ is $\geq \max \left(\bar{c}_{i}\right) \geq \operatorname{deg}(u)$. We delete those $s$ vertices in $B$, and connect $u$ to each of $v_{1}, \ldots, v_{s}$ by $E_{i}$-edges. We do this until the set $B$ is empty. Similarly, by Inequality 3 , we can perform similar operations until the set $A$ is empty. The resulting graph is a $(C, D)$-biregular graph of size $(\bar{M}, \bar{N})$. This completes the proof of Lemma 7.5 .

Now Lemma 7.5 tells us the Presburger formula BiREG ${ }_{C, D}$ for a pair of matrices satisfying the assumption given in Lemma 7.5. More formally, let $C \in\left(\begin{array}{c}C^{(1)} \\ C^{(2)} \\ C^{(3)}\end{array}\right) \in \mathbb{B}^{\ell \times m}$ and $D \in\left(\begin{array}{c}D^{(1)} \\ D^{(2)} \\ D^{(3)}\end{array}\right) \in$ $\mathbb{B}^{\ell \times n}$ where $\ell=\ell_{1}+\ell_{2}+\ell_{3}$ and

- $C^{(1)} \in \mathbb{N}^{\ell_{1} \times m}$ and $D^{(1)} \in \mathbb{N}^{\ell_{1} \times n}$;

- $C^{(2)} \in \mathbb{N}^{\ell_{2} \times m}$ and $D^{(2)} \in \mathbb{B}^{\ell_{2} \times n}$ and every row in $D^{(2)}$ contains an element of $\bullet \mathbb{N}$;

- $C^{(3)} \in \mathbb{B}^{\ell_{3} \times m}$ and $D^{(3)} \in \mathbb{N}^{\ell_{3} \times n}$ and every row in $C^{(3)}$ contains an element of $\bullet \mathbb{N}$.

That is, for such $C, D$, we let $\operatorname{BiREG}_{C, D}(\bar{X}, \bar{Y})$ as follows.

$$
\begin{aligned}
\operatorname{BiREG}_{C, D}(\bar{X}, \bar{Y}):=\quad \exists Z_{1} \cdots & \exists Z_{\ell_{3}} \exists Z_{1}^{\prime} \cdots \exists Z_{\ell_{2}}^{\prime} \\
& \operatorname{BiREG}_{C^{\prime}, D^{\prime}}\left(\bar{X}, Z_{1}, \ldots, Z_{\ell_{3}}, \bar{Y}, Z_{1}^{\prime}, \ldots, Z_{\ell_{2}}^{\prime}\right)
\end{aligned}
$$

where $C^{\prime}=\left(\begin{array}{c|c}C^{(1)} & 0 \\ \hline C^{(2)} & 0 \\ \hline\left\lfloor C^{(3)}\right\rfloor & I_{\ell_{3}}\end{array}\right) \in \mathbb{N}^{\ell \times\left(m+\ell_{3}\right)}$ and $D^{\prime}=\left(\begin{array}{c|c}D^{(1)} & 0 \\ \hline\left\lfloor D^{(2)}\right\rfloor & I_{\ell_{2}} \\ \hline D^{(3)} & 0\end{array}\right) \in \mathbb{N}^{\ell \times\left(n+\ell_{2}\right)}$. Since $C^{\prime}, D^{\prime}$ consist of entirely $\mathbb{N}$ entries, BiREG $C_{C^{\prime}, D^{\prime}}$ is defined as in Theorem 7.4. Intuitively, the variables 
$Z_{1}, \ldots, Z_{\ell_{3}}$ are to capture the values $K_{1}, \ldots, K_{\ell_{3}}$ and $Z_{1}^{\prime}, \ldots, Z_{\ell_{2}}$ the values $L_{1}, \ldots, L_{\ell_{2}}$, as stated in Lemma 7.5 .

Using this, we can prove the following theorem.

Theorem 7.6 For every $C \in \mathbb{B}^{\ell \times m}$ and $D \in \mathbb{B}^{\ell \times n}$, there is a Presburger formula $\widetilde{\operatorname{BiRE} G_{C, D}}(\bar{X}, \bar{Y})$ such that for every $\bar{M}, \bar{N}$ big enough with respect to $C, D$, the following holds. There exists a $(C, D)$-biregular graph of size $(\bar{M}, \bar{N})$ if and only if the statement $\widetilde{\operatorname{BiREG}}_{C, D}(\bar{M}, \bar{N})$ holds.

Proof. Let $C \in \mathbb{B}^{\ell \times m}$ and $D \in \mathbb{B}^{\ell \times n}$, where $\bar{c}_{1}, \ldots, \bar{c}_{\ell}$ and $\bar{d}_{1}, \ldots, \bar{d}_{\ell}$ are the row vectors of $C$ and $D$, respectively.

We need an additional notation. For a set $I \subseteq\{1, \ldots, \ell\}$, we write $C(I)$ be the matrix $C^{\prime}$, in which each row vector $\bar{c}_{i}^{\prime}$ is defined as $\bar{c}_{i}^{\prime}=\left\lfloor\bar{c}_{i}\right\rfloor$, if $i \in I$, and $\bar{c}_{i}^{\prime}=\bar{c}_{i}$, if $i \notin I$. We can define $D(I)$ similarly.

We define the formula $\operatorname{BiREG}_{C, D}(\bar{X}, \bar{Y})$ as follows.

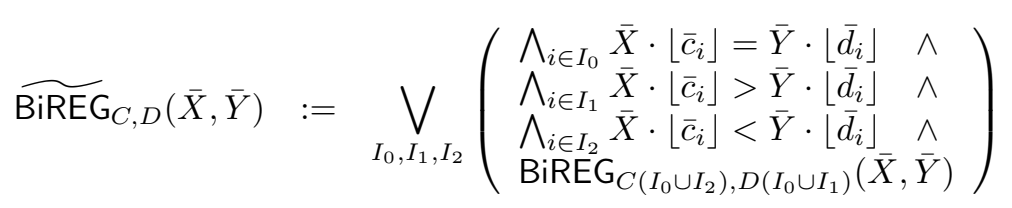

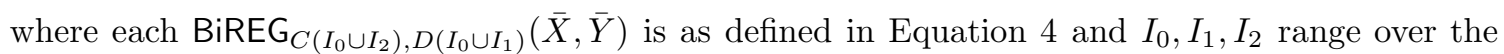
partition $I_{0} \cup I_{1} \cup I_{2}=\{1,2, \ldots, \ell\}$. Obviously, for every partition $I_{0} \cup I_{1} \cup I_{2}=\{1, \ldots, \ell\}$, on each row $i=1, \ldots, \ell$, either row- $i$ from $C\left(I_{0} \cup I_{2}\right)$, or row- $i$ from $D\left(I_{0} \cup I_{1}\right)$, or row- $i$ from both consists entirely of $\mathbb{N}$. (Our intention is the application of Lemma 7.5 later on.)

We are going to prove that the formula $\widetilde{\operatorname{BiREG}}_{C, D}(\bar{X}, \bar{Y})$ is the desired formula. The "if" direction follows from Lemma 7.5 and that every $C\left(I_{0} \cup I_{2}\right), D\left(I_{0} \cup I_{1}\right)$-biregular graph is obviously also a $(C, D)$-biregular graph.

Now we prove the "only if" direction. Suppose $\bar{M}, \bar{N}$ are big enough for $C, D$. Let $G=$ $\left(U, V, E_{1}, \ldots, E_{\ell}\right)$ be a $(C, D)$-biregular graph of size $(\bar{M}, \bar{N})$, where $U=U_{1} \cup \cdots \cup U_{m}$ and $V=$ $V_{1} \cup \cdots \cup V_{n}$ be the witness of the $C, D$-biregularity.

We pick the following paritition $I_{0} \cup I_{1} \cup I_{2}=\{1, \ldots, \ell\}$.

$$
\begin{aligned}
& I_{0}=\left\{i \mid \bar{M} \cdot\left\lfloor\bar{c}_{i}\right\rfloor=\bar{M} \cdot\left\lfloor\bar{d}_{i}\right\rfloor\right\} \\
& I_{1}=\left\{i \mid \bar{M} \cdot\left\lfloor\bar{c}_{i}\right\rfloor>\bar{M} \cdot\left\lfloor\bar{d}_{i}\right\rfloor\right\} \\
& I_{2}=\left\{i \mid \bar{M} \cdot\left\lfloor\bar{c}_{i}\right\rfloor<\bar{M} \cdot\left\lfloor\bar{d}_{i}\right\rfloor\right\}
\end{aligned}
$$

We are going to convert the graph $G$ into $\left(C\left(I_{0} \cup I_{1}\right), D\left(I_{0}, I_{2}\right)\right)$-biregular graph in which $U=$ $U_{1} \cup \cdots \cup U_{m}$ and $V=V_{1} \cup \cdots \cup V_{n}$ are also the witness of the $\left(C\left(I_{0} \cup I_{1}\right), D\left(I_{0}, I_{2}\right)\right)$-biregularity. This, together with Lemma 7.5. implies that $\operatorname{BiREG}_{C\left(I_{0} \cup I_{1}\right), D\left(I_{0}, I_{2}\right)}(\bar{M}, \bar{N})$ holds, and hence, our theorem.

If $G$ is already a $\left(C\left(I_{0} \cup I_{1}\right), D\left(I_{0}, I_{2}\right)\right)$-biregular graph, then we are done. Suppose that $G$ is not. We do the following three stages.

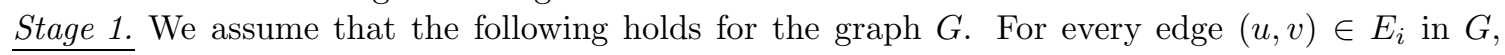
either

$$
\operatorname{deg}_{E_{i}}(u)=\left\lfloor C_{i, j}\right\rfloor \quad \text { or } \quad \operatorname{deg}_{E_{i}}(v)=\left\lfloor D_{i, k}\right\rfloor .
$$

This can be achieved by doing the following. Suppose there is an edge $(u, v) \in E_{i}$ such that $\operatorname{deg}_{E_{i}}(u)>\left\lfloor C_{i, j}\right\rfloor$ and $\operatorname{deg}_{E_{i}}(v)>\left\lfloor D_{i, k}\right\rfloor$. Since $G$ is $(C, D)$-biregular, this means that $C_{i, j}, D_{i, k} \in$ $\rightarrow \mathbb{N}$.

Deleting the edge $(u, v)$, we still have $\operatorname{deg}_{E_{i}}(u) \geq\left\lfloor C_{i, j}\right\rfloor$ and $\operatorname{deg}_{E_{i}}(v) \geq\left\lfloor D_{i, k}\right\rfloor$, and hence $G$ is still $(C, D)$-biregular with $U=U_{1} \cup \cdots \cup U_{m}$ and $V=V_{1} \cup \cdots \cup V_{n}$ be the witness of the $(C, D)$-biregularity. We repeatedly do this until the graph $G$ satisfies condition (5).

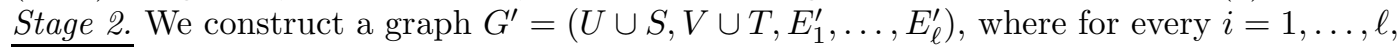

- for every $j=1, \ldots, m$, for each $u \in U_{j}, \operatorname{deg}_{E_{i}^{\prime}}(u)=\left\lfloor C_{i, j}\right\rfloor$, 
- for every $s \in S, \operatorname{deg}(s)=1$ 泟

- for every $k=1, \ldots, n$, for each $v \in V_{j}, \operatorname{deg}_{E_{i}^{\prime}}(v)=\left\lfloor D_{i, j}\right\rfloor$,

- for every $t \in T, \operatorname{deg}(t)=1$.

The graph $G^{\prime}$ can be obtained by doing the same trick as in the proof of Lemma 7.5. For every vertex $u \in U_{j}$, if $\operatorname{deg}_{E_{i}}(u)-\left\lfloor C_{i, j}\right\rfloor=z>0$, then we "split" $u$ into $z+1$ vertices $u^{\prime}, s_{1}, \ldots, s_{z}$, where

- $\operatorname{deg}_{E_{i}}\left(u^{\prime}\right)=\left\lfloor C_{i, j}\right\rfloor$, for all other $h \neq i, \operatorname{deg}_{E_{h}}\left(u^{\prime}\right)=\operatorname{deg}_{E_{h}}(u)$;

- $\operatorname{deg}_{E_{i}}\left(s_{1}\right)=\cdots=\operatorname{deg}_{E_{i}}\left(s_{z}\right)=1$, and for all other $h \neq i, \operatorname{deg}_{E_{h}}\left(s_{1}\right)=\cdots=\operatorname{deg}_{E_{h}}\left(s_{z}\right)=0$.

We can do similar operation to the vertices in $v \in V_{k}$. Since $G$ satisfies condition 5 , there is no edge between vertices in $S$ and $T$. We also further partition $S=S_{1} \cup \cdots \cup S_{\ell}$ and $T=T_{1} \cup \cdots \cup T_{\ell}$, where each $S_{i}$ and $T_{i}$ contains the vertices whose $\operatorname{deg}_{E_{i}}=1$.

Stage 3. Stage 3 is as follows. For each $i=1, \ldots, \ell$, if there are an edge $(s, v) \in E_{i}$ and an edge $\overline{(u, t) \in E_{i}}$, for some $s \in S_{i}, v \in V_{k}, u \in U_{j}, t \in T_{i}$, we do the following.

- We delete the two edges $(s, v)$ and $(u, t)$ from $E_{i}$, as well as the vertices $s$ and $t$.

- We add an edge $(u, v)$ into $E_{i}$.

- If there is already an existing edge $(u, v) \in E_{1} \cup \cdots \cup E_{\ell}$, adding another $(u, v)$ may result in "parallel" edges. However, since $\bar{M}, \bar{N}$ is big enough with respect to $C, D$, and in particular, Inequality 1 holds, we can apply the same trick as in the proof of Theorem 7.4 to get rid of the parallel edge, while preserving the degree of the vertices.

We repeatedly do this until for each $i=1, \ldots, \ell$ either $S_{i}=\emptyset$, or $T_{i}=\emptyset$. In particular, the following holds.

- If $i \in I_{0}$, then $S_{i}=T_{i}=0$.

Recall that $i \in I_{0}$ means that $\bar{M} \cdot\left\lfloor\bar{c}_{i}\right\rfloor=\bar{N} \cdot\left\lfloor\bar{d}_{i}\right\rfloor$, which implies that the initial sets $S_{i}, T_{i}$ have the same cardinality. Since we always delete a pair of vertices $s, t$ from $S_{i}, T_{i}$, respectively, we have at the end $S_{i}=T_{i}=\emptyset$.

- Likewise, if $i \in I_{1}$, then $S_{i}=0$.

This is because $\bar{M} \cdot\left\lfloor\bar{c}_{i}\right\rfloor>\bar{N} \cdot\left\lfloor\bar{d}_{i}\right\rfloor$, implies that initially $\left|T_{i}\right|>\left|S_{i}\right|$, which further implies that at the end $S_{i}=\emptyset$.

By symmetrical reasoning, if $i \in I_{2}$, then $T_{i}=0$.

From here, we will "merge" back the vertices in $T$ with vertices in $V$. This is done as follows. For each vertex $u \in U$ adjacent by $E_{i}$-edges to, say, $z$ vertices in $T$, we pick $z$ vertices $v_{1}, \ldots, v_{z}$ from the set

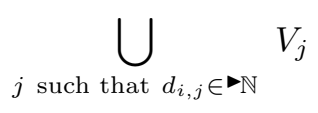

Such $z$ vertices exist by Inequality 2 (because $\bar{M}, \bar{N}$ are big enough w.r.t. $C, D$ ). We delete those $z$ vertices in $T$, and connect $u$ to each of $v_{1}, \ldots, v_{z}$ by $E_{i}$-edges. We do this until the set $T$ is empty. In a similar manner, we can merge back the vertices in $S$ with vertices in $U$, where the existence of the vertices $v_{1}, \ldots, v_{z}$ is guaranteed by Inequality 3 ,

The resulting graph is $\left(C\left(I_{0} \cup I_{1}\right), D\left(I_{0}, I_{2}\right)\right)$-biregular graph, which by Lemma 7.5 the formula implies that $\operatorname{BiREG}_{C\left(I_{0} \cup I_{2}\right), D\left(I_{0} \cup I_{1}\right)}(\bar{M}, \bar{N})$ holds. This completes our proof of Theorem 7.6 ,

\footnotetext{
$s$.

${ }^{* *}$ Recall that $\operatorname{deg}(s)=\operatorname{deg}_{E_{1}^{\prime}}(s)+\cdots \operatorname{deg}_{E_{\ell}^{\prime}}(s)$. Hence, $\operatorname{deg}(s)=1$ means that there is only one edge adjacent to
} 


\subsection{The notion of partial bipartite graphs}

In this subsection we are going to generalise Theorem 7.6 to the case when it is possible that one of the inequalities 1 and 2 does not hold. The idea is that those numbers (for which the inequalities do not hold) are hard coded into the Presburger formula. For this, we introduce the notion of partial graph.

An $\ell$-type partial bipartite graph is a tuple $\mathcal{P}=(C, D, S, T, f, g)$, where

- $C \in \mathbb{B}^{\ell \times m}$ and $D \in \mathbb{B}^{\ell \times n}$,

- $S$ is a finite set of vertices (possibly empty),

- $T$ is a finite set of vertices (possibly empty),

- $f: S \times\left\{E_{1}, \ldots, E_{\ell}\right\} \rightarrow \mathbb{B}$

- $g: T \times\left\{E_{1}, \ldots, E_{\ell}\right\} \rightarrow \mathbb{B}$.

Obviously, if $S$ or $T$ is empty, then $f$ or $g$, respectively, is also an "empty" function. In the following the term partial graph always means partial bipartite graph.

A completion of the partial graph $\mathcal{P}=(C, D, S, T, f, g)$ is a bipartite graph $G=(U \cup S, V \cup$ $\left.T, E_{1}, \ldots, E_{\ell}\right)$ such that there is a partition $U_{1} \cup \cdots \cup U_{m}$ of $U$ and a partition $V_{1} \cup \cdots \cup V_{n}$ of $V$ such that

- for every $u \in U_{j}, \operatorname{deg}_{E_{i}}(u)=C_{i, j}$,

- for every $v \in V_{j}, \operatorname{deg}_{E_{i}}(v)=D_{i, j}$,

- for every $s \in S, \operatorname{deg}_{E_{i}}(s)=f\left(s, E_{i}\right)$,

- for every $t \in T, \operatorname{deg}_{E_{i}}(t)=g\left(t, E_{i}\right)$.

When it is clear from the context, we also call $U=U_{1} \cup \cdots \cup U_{m}$ and $V=V_{1} \cup \cdots \cup V_{n}$ the witness of the $(C, D)$-biregularity. Note that when both $S$ and $T$ are empty, then the completions of the partial graph $\mathcal{P}$ are simply $(C, D)$-biregular graphs.

We need a few additional notations.

$$
\left(\bullet_{c}\right)-d= \begin{cases}\nabla_{(}(c-d) & \text { if } c \geq d \\ \nabla_{0} & \text { otherwise }\end{cases}
$$

Let $C \in \mathbb{B}^{\ell \times m}$. We define a matrix $\xi(C) \in \mathbb{B}^{\ell \times(\ell+1) m}$ as follows.

$$
\xi(C):=\left(C\left|M_{1}\right| \cdots \mid M_{m}\right)
$$

where each $M_{i}$ is the matrix obtained by repeating the $i$ th column vector of $C$ for $\ell$ number of times, and substracting the identity matrix $\mathbf{I}_{\ell}$. Formally,

$$
M_{i}:=\left(\begin{array}{cccc}
C_{1, i} & C_{1, i} & \cdots & C_{1, i} \\
C_{2, i} & C_{2, i} & \cdots & C_{2, i} \\
\vdots & \vdots & \ddots & \vdots \\
C_{\ell, i} & C_{\ell, i} & \cdots & C_{\ell, i}
\end{array}\right)-\mathbf{I}_{\ell}
$$

Lemma 7.7 below essentially states that every partial graph can be reduced into a "smaller" partial graph with the addition of some linear equalities.

Lemma 7.7 Let $\mathcal{P}=(C, D, S, T, f, g)$ be a partial graph, where $T \neq \emptyset$. Let $t \in T$. Then the following holds.

(1) For every completion graph $G=\left(U \cup S, V \cup T, E_{1}, \ldots, E_{\ell}\right)$ of the partial graph $\mathcal{P}$ with $U=$ $U_{1} \cup \cdots \cup U_{m}$ and $V=V_{1} \cup \cdots \cup V_{n}$ being the witness of the $(C, D)$-biregularity, there exists a 
completion graph $G^{\prime}=\left(U \cup S,(V \cup T) \backslash\{t\}, E_{1}^{\prime}, \ldots, E_{\ell}^{\prime}\right)$ of the partial graph $\mathcal{P}^{\prime}=(\xi(C), D, S, T \backslash$ $\left.\{t\}, f, g^{\prime}\right)$, with the witness of the $(\xi(C), D)$-biregularity being

$$
\begin{aligned}
U= & U_{1}^{\prime} \cup \cdots \cup U_{m}^{\prime} \cup \\
& \left(U_{1,1}^{\prime} \cup \cdots \cup U_{\ell, 1}^{\prime}\right) \cup\left(U_{1,2}^{\prime} \cup \cdots \cup U_{\ell, 2}^{\prime}\right) \cup \cdots \cup\left(U_{1, m}^{\prime} \cup \cdots \cup U_{\ell, m}^{\prime}\right) \\
V= & V_{1} \cup \cdots \cup V_{n}
\end{aligned}
$$

and

$$
\begin{aligned}
\left|U_{j}\right| & =\left|U_{j}^{\prime}\right|+\left|U_{1, j}^{\prime}\right|+\cdots+\left|U_{\ell, j}^{\prime}\right| & & \text { for each } j=1, \ldots, m \\
\sum_{1 \leq j \leq m}\left|U_{i, j}^{\prime}\right| & =g\left(t, E_{i}\right) & & \text { for each } i=1, \ldots, \ell .
\end{aligned}
$$

(2) Visa versa, for every completion graph $G^{\prime}=\left(U \cup S,(V \cup T) \backslash\{t\}, E_{1}^{\prime}, \ldots, E_{\ell}^{\prime}\right)$ of the partial graph $\mathcal{P}^{\prime}=\left(\xi(C), D, S, T \backslash\{t\}, f, g^{\prime}\right)$, with the witness of the $(\xi(C), D)$-biregularity being

$$
\begin{aligned}
U= & U_{1}^{\prime} \cup \cdots \cup U_{m}^{\prime} \cup \\
& \left(U_{1,1}^{\prime} \cup \cdots \cup U_{\ell, 1}^{\prime}\right) \cup\left(U_{1,2}^{\prime} \cup \cdots \cup U_{\ell, 2}^{\prime}\right) \cup \cdots \cup\left(U_{1, m}^{\prime} \cup \cdots \cup U_{\ell, m}^{\prime}\right) \\
V= & V_{1} \cup \cdots \cup V_{n}
\end{aligned}
$$

and for each $i=1, \ldots, \ell, \sum_{1 \leq j \leq m}\left|U_{i, j}^{\prime}\right|=g\left(t, E_{i}\right)$, there exists a completion graph $G=$ $\left(U \cup S, V \cup T, E_{1}, \ldots, E_{\ell}\right)$ of the partial graph $\mathcal{P}$ with $U=U_{1} \cup \cdots \cup U_{m}$ and $V=V_{1} \cup \cdots \cup V_{n}$ being the witness of the $(C, D)$-biregularity, and

$$
\left|U_{j}\right|=\left|U_{j}^{\prime}\right|+\left|U_{1, j}^{\prime}\right|+\cdots+\left|U_{\ell, j}^{\prime}\right| \quad \text { for each } j=1, \ldots, m .
$$

Proof. Let $\mathcal{P}=(C, D, S, T, f, g)$ be a partial graph, where $T \neq \emptyset$ and $t \in T$. First, we prove part (1). Let $G=\left(U \cup S, V \cup T, E_{1}, \ldots, E_{\ell}\right)$ be a completion graph of $\mathcal{P}$ with $U=U_{1} \cup \cdots \cup U_{m}$ and $V=V_{1} \cup \cdots \cup V_{n}$ being the witness of the $(C, D)$-biregularity.

For each $j=1, \ldots, m$, we partition $U_{j}$ into

$$
U_{j}=U_{j}^{\prime} \cup\left(U_{1, j}^{\prime} \cup \cdots \cup U_{\ell, j}^{\prime}\right),
$$

where

- $U_{j}^{\prime}$ be the set of vertices in $U_{j}$ that are not adjacent to the vertex $t$,

- for each $i=1, \ldots, \ell, U_{i, j}^{\prime}$ is the set of vertices in $U_{j}$ adjacent to $t$ via $E_{i}$-edges.

Now deleting the vertex $t$ and all its adjacent edges, we obtain the desired completion graph $G^{\prime}=\left(U \cup S,(V \cup T) \backslash\{t\}, E_{1}^{\prime}, \ldots, E_{\ell}^{\prime}\right)$ of $\mathcal{P}^{\prime}=\left(\xi(C), D, S, T \backslash\{t\}, f, g^{\prime}\right)$.

Now we prove part (2). Let $G^{\prime}=\left(U \cup S,(V \cup T) \backslash\{t\}, E_{1}^{\prime}, \ldots, E_{\ell}^{\prime}\right)$ be a completion of the partial graph $\mathcal{P}^{\prime}=\left(\xi(C), D, S, T \backslash\{t\}, f, g^{\prime}\right)$, with the witness of the $(\xi(C), D)$-biregularity being

$$
\begin{aligned}
U= & U_{1}^{\prime} \cup \cdots \cup U_{m}^{\prime} \cup \\
& \left(U_{1,1}^{\prime} \cup \cdots \cup U_{\ell, 1}^{\prime}\right) \cup\left(U_{1,2}^{\prime} \cup \cdots \cup U_{\ell, 2}^{\prime}\right) \cup \cdots \cup\left(U_{1, m}^{\prime} \cup \cdots \cup U_{\ell, m}^{\prime}\right) \\
V= & V_{1} \cup \cdots \cup V_{n}
\end{aligned}
$$

and for each $i=1, \ldots, \ell, \sum_{1 \leq j \leq m}\left|U_{i, j}^{\prime}\right|=g\left(t, E_{i}\right)$.

The desired completion graph $G=\left(U \cup S, V \cup T, E_{1}, \ldots, E_{\ell}\right)$ of the partial graph $\mathcal{P}$ can be obtained as follows. We put the vertex $t$ back inside $T$. Then, for each $i=1, \ldots, \ell$ and for each $j=1, \ldots, m$, we connect $t$ with every vertex $u \in U_{i, j}^{\prime}$ with $E_{i}$-edge. This way we obtain the completion graph $G$ with $U=U_{1} \cup \cdots \cup U_{m}$ and $V=V_{1} \cup \cdots \cup V_{n}$ being the witness of the $(C, D)$-biregularity, and

$$
\left|U_{i}\right|=\left|U_{i}^{\prime}\right|+\left|U_{1, i}^{\prime}\right|+\cdots+\left|U_{\ell, i}^{\prime}\right| \quad \text { for each } i=1, \ldots, m .
$$


This completes our proof of Lemma 7.7

Following Lemma 7.7 above, we show that every partial graph can be translated into a Presburger formula that captures any of its completion, as stated in the following theorem.

Theorem 7.8 For every partial graph $\mathcal{P}=(C, D, S, T, f, g)$, we can construct a Presburger formula $\Psi_{\mathcal{P}}(\bar{X}, \bar{Y})$ such that for every $\bar{M}$ and $\bar{N}$ big enough w.r.t. $C, D$, the following holds. There exists a completion graph $G=\left(U \cup S, V \cup T, E_{1}, \ldots, E_{\ell}\right)$, such that $U=U_{1} \cup \cdots \cup U_{m}$ and $V=V_{1} \cup \cdots \cup V_{n}$ and $\bar{M}=\left(\left|U_{1}\right|, \ldots,\left|U_{m}\right|\right)$ and $\bar{N}=\left(\left|V_{1}\right|, \ldots,\left|V_{n}\right|\right)$ if and only if $\Psi_{\mathcal{P}}(\bar{M}, \bar{N})$ holds.

Proof. Let $\mathcal{P}=(C, D, S, T, f, g)$ be a partial graph. If the matrix $C$ is empty, there are only finitely many completion of $\mathcal{P}$. In this case $\Psi_{\mathcal{P}}$ simply contains the enumeration the sizes of all possible completions of $\mathcal{P}$. We can define $\Psi_{\mathcal{P}}$ in a similar manner when $D$ is empty.

Now suppose both the matrices $C$ and $D$ are not empty. The construction of $\Psi_{\mathcal{P}}$ is done inductively as follows. The base case is $S \cup T=\emptyset$, in which case $\Psi_{\mathcal{P}}$ is defined as follows.

$$
\Psi_{\mathcal{P}}(\bar{X}, \bar{Y}):=\widetilde{\operatorname{BiREG}}_{C, D}(\bar{X}, \bar{Y})
$$

where $\widetilde{\operatorname{BiREG}}_{C, D}(\bar{X}, \bar{Y})$ is as defined in Theorem 7.6 .

Towards the induction step, let $S \cup T \neq \emptyset$. Suppose $T \neq \emptyset$ and $t \in T$. (The case when $S \neq \emptyset$ can handled in a symmetrical manner.)

We define $\Psi_{\mathcal{P}}(\bar{X}, \bar{Y})$ as follows.

$$
\begin{aligned}
& \Psi_{\mathcal{P}}(\bar{X}, \bar{Y}):=\exists Z_{1} \cdots \exists Z_{m} \exists Z_{1,1} \cdots \exists Z_{\ell, 1} \exists Z_{1,2} \cdots \exists Z_{\ell, 2} \cdots \exists Z_{1, m} \cdots \exists Z_{\ell, m} \\
& \wedge \bigwedge_{1 \leq i \leq m} X_{i}=Z_{i}+Z_{1, i}+\cdots+Z_{\ell, i} \\
& \wedge \bigwedge_{1 \leq i \leq \ell} \sum_{1 \leq j \leq m} Z_{i, j}=g\left(t, E_{i}\right) \\
& \wedge \quad \Psi_{\mathcal{P}^{\prime}}\left(\left(Z_{1}, \ldots, Z_{m}, Z_{1,1}, \ldots, Z_{\ell, 1}, \ldots, Z_{1, m}, \ldots, Z_{\ell, m}\right), \bar{Y}\right)
\end{aligned}
$$

where $\mathcal{P}^{\prime}=\left(\xi(C), D, S, T \backslash\{t\}, f, g^{\prime}\right)$ and $g^{\prime}$ is the function $g$ restricted to $T \backslash\{t\}$.

By Theorem [7.6 in the previous section, the correctness of the base case is established. The induction step follows from Lemma 7.7, and hence, shows that the formula $\Psi_{\mathcal{P}}$ is the desired formula. This completes our proof of Theorem 7.8 .

\subsection{Constructing the formula $\operatorname{BiREG}_{C, D}(\bar{X}, \bar{Y})$ for Theorem 5.1}

We need the following notions. Let $C \in \mathbb{B}^{\ell \times m}$ and $D \in \mathbb{B}^{\ell \times n}$. We say that a partial graph $\mathcal{P}=\left(C^{\prime}, D^{\prime}, S, T, f, g\right)$ is compatible with $(C, D)$ with respect to a subset $I \subseteq\{1, \ldots, m\}$ and a subset $J \subseteq\{1, \ldots, n\}$, and the partitions $S=S_{1} \cup \cdots \cup S_{m^{\prime \prime}}$ and $T=T_{1} \cup \cdots \cup T_{n^{\prime \prime}}$, if the following four conditions hold.

- $C^{\prime} \in \mathbb{B}^{\ell \times m^{\prime}}$ is obtained by deleting the columns $I$ in $C$.

- $D^{\prime} \in \mathbb{B}^{\ell \times n^{\prime}}$ is obtained by deleting the columns $J$ in $D$.

- Let $C^{\prime \prime} \in \mathbb{B}^{\ell \times m^{\prime \prime}}$ be the matrix whose columns are the columns $I$ in $C$ where $m^{\prime \prime}=m-m^{\prime}$. The matrix $C^{\prime \prime}$ is simply the matrix form of the function $f$. That is, for each $k=1, \ldots, m^{\prime \prime}$, for every vertex $s \in S_{k}$, for every $i=1, \ldots, \ell, f\left(s, E_{i}\right)=C_{i, k}^{\prime \prime}$.

- Let $D^{\prime \prime} \in \mathbb{B}^{\ell \times n^{\prime \prime}}$ be the matrix whose columns are the columns $J$ in $D$ where $n^{\prime \prime}=n-n^{\prime}$. The matrix $D^{\prime \prime}$ is simply the matrix form of the function $g$. That is, for each $k=1, \ldots, n^{\prime \prime}$, for every vertex $t \in T_{k}$, for every $i=1, \ldots, \ell, g\left(t, E_{i}\right)=D_{i, k}^{\prime \prime}$.

For a subset $I \subseteq\{1, \ldots, m\}$, and a variable vector $\bar{X}=\left(X_{1}, \ldots, X_{\ell}\right)$, we write $\bar{X}_{I}$ to denote the variables obtained by deleting $X_{i}$ whenever $i \in I$. We can define $\bar{Y}_{J}$ similarly when $J \subseteq\{1, \ldots, n\}$ and $\bar{Y}=\left(Y_{1}, \ldots, Y_{n}\right)$. 
The formula $\operatorname{BiREG}_{C, D}(\bar{X}, \bar{Y})$ as as required in Theorem 5.1 is as follows.

$$
\operatorname{BiREG}_{C, D}(\bar{X}, \bar{Y}):=\bigvee_{\mathcal{P}}\left(\begin{array}{l}
\Psi_{\mathcal{P}}\left(\bar{X}_{I}, \bar{Y}_{J}\right) \wedge \varphi \\
\wedge X_{i_{1}}=\left|S_{1}\right| \wedge X_{i_{2}}=\left|S_{2}\right| \wedge \cdots \wedge \wedge X_{i_{m^{\prime \prime}}}=\left|S_{m^{\prime \prime}}\right| \\
\wedge Y_{j_{1}}=\left|T_{1}\right| \wedge Y_{j_{2}}=\left|t_{2}\right| \wedge \cdots \wedge \wedge Y_{j_{n^{\prime \prime}}}=\left|T_{n^{\prime \prime}}\right|
\end{array}\right)
$$

where

- the disjunction ranges over all partial graph $\mathcal{P}=\left(C^{\prime}, D^{\prime}, S, T, f, g\right)$ compatible with $(C, D)$ w.r.t. $I=\left\{i_{1}, \ldots, i_{m^{\prime \prime}}\right\}$ and $J=\left\{j_{1}, \ldots, j_{n^{\prime \prime}}\right\}$, as well as the partitions $S=S_{1} \cup \cdots \cup S_{m^{\prime \prime}}$ and $T=T_{1} \cup \cdots \cup T_{n^{\prime \prime}}$,

- the formula $\psi_{\mathcal{P}}$ is as defined in Theorem 7.8

- $\varphi$ states that $\bar{X}_{I}, \bar{Y}_{J}$ are big enough w.r.t. $\left(C^{\prime}, D^{\prime}\right)$, as defined in the Inequalities (1), (2) and (3).

The correctness of the formula $\mathrm{BiREG}_{C, D}$ follows immediately from the correctness of the formula $\Psi_{\mathcal{P}}$ in Theorem 7.8. This completes our proof of Theorem 5.1.

\subsection{Constructing the formula COMP-BiREG $\operatorname{CO}_{C, D}(\bar{X}, \bar{Y})$ for Theorem 5.2}

We start with Lemma 7.9 which essentially states that if there exists a $(C, D)$-biregular-complete graph of "big enough" size, then for every column $i$ in $C$ and every column $j$ in $D$, there is a row $l$ such that both $C_{l, i}, D_{l, j} \in \mathbb{N}$. This means that a $(C, D)$-biregular-complete graph $G=$ $\left(U, V, E_{1}, \ldots, E_{\ell}\right)$ of "big enough" size $(\bar{M}, \bar{N})$, then we can connect every pair of vertices $u \in U$ and $v \in V$ with one of the edges without violating the $(C, D)$-biregularity.

Lemma 7.9 Let $G=\left(U, V, E_{1}, \ldots, E_{\ell}\right)$ be an $\ell$-type $(C, D)$-biregular graph and $U=U_{1} \cup \cdots \cup U_{m}$ and $V=V_{1} \cup \cdots \cup V_{n}$ be the witness of the $(C, D)$-biregularity. Suppose that for each $i, j$, we have

$$
\left|U_{i}\right|, \quad\left|V_{j}\right| \geq\lfloor C\rfloor \cdot \overline{1}+\lfloor D\rfloor \cdot \overline{1}+1 .
$$

If $G$ is a complete bipartite graph, then for every $i \in\{1, \ldots, m\}$ and $j \in\{1, \ldots, n\}$, there exists $l \in\{1, \ldots, \ell\}$ such that both $C_{l, i}, D_{l, j} \in \mathbb{N}$.

Proof. Let $C \in \mathbb{B}^{\ell \times m}$ and $D \in \mathbb{B}^{\ell \times n}$, and $G=\left(U, V, E_{1}, \ldots, E_{\ell}\right)$ be a $(C, D)$-biregular-complete graph, where $U=U_{1} \cup \cdots \cup U_{n}$ and $V=V_{1} \cup \cdots \cup V_{n}$ are the witness of the $(C, D)$-biregularity. Suppose each $\left|U_{i}\right|$ and $\left|V_{j}\right|$ satisfy the inequality above.

For the sake of contradiction, we assume that that there exist $i, j \in\{1, \ldots, m\}$ such that for all $l \in\{1, \ldots, \ell\}$, either $C_{l, i} \in \mathbb{N}$ or $D_{l, j} \in \mathbb{N}$. This means that for each $l \in\{1, \ldots, \ell\}$, the number of $E_{l}$-edges between $U_{i}$ and $V_{j}$ is $\left|U_{i}\right| C_{l, i}$, if $C_{l, i} \in \mathbb{N}$, or $\left|V_{j}\right| D_{l, j}$, if $D_{l, j} \in \mathbb{N}$. For each $l=1, \ldots, \ell$,

$$
K_{l}= \begin{cases}\left|U_{i}\right| C_{l, i} & \text { if } C_{l, i} \in \mathbb{N} \\ \left|V_{j}\right| D_{l, j} & \text { if } D_{l, j} \in \mathbb{N}\end{cases}
$$

Now the total number of edges between $U_{i}$ and $V_{j}$ must be $\sum_{1 \leq l \leq \ell} K_{l}$, which must be equal to $\left|U_{i}\right| \times\left|V_{j}\right|$ since $G$ is a complete bipartite graph.

However, from the inequality

$$
\left|U_{i}\right|, \quad\left|V_{j}\right| \geq\lfloor C\rfloor \cdot \overline{1}+\lfloor D\rfloor \cdot \overline{1}+1,
$$

a straightforward calculation shows that $K$ is strictly less than $\left|U_{i}\right| \times\left|V_{j}\right|$, a contradiction. Therefore, for every $i \in\{1, \ldots, m\}$ and $j \in\{1, \ldots, n\}$, there exists $l \in\{1, \ldots, \ell\}$ such that both $C_{l, i}, D_{l, j} \in \mathbb{N}$. This completes the proof of our lemma.

We say that a pair of matrices $(C, D) \in \mathbb{B}^{\ell \times m} \times \mathbb{B}^{\ell \times n}$ is an easy pair of matrices, if for every $i \in\{1, \ldots, m\}$ and $j \in\{1, \ldots, n\}$ there exists $l \in\{1, \ldots, \ell\}$ such that both $C_{l, i}, D_{l, j} \in \mathbb{N}$.

Lemma 7.10 says that if $(C, D)$ is an easy pair of matrices, then the formula $\operatorname{BiREG}_{C, D}(\bar{X}, \bar{Y})$ as defined in Subsection 7.5 is sufficient as the required formula $\operatorname{COMP-BiREG}_{C, D}(\bar{X}, \bar{Y})$ in Theorem 5.2 , 
Lemma 7.10 Let $(C, D)$ be an easy pair of matrices, where $C \in \mathbb{B}^{\ell \times m}$ and $D \in \mathbb{B}^{\ell \times n}$. Then the following holds. There exists a $(C, D)$-biregular-complete graph of size $(\bar{M}, \bar{N})$ if and only if $\operatorname{BiRE}_{C, D}(\bar{M}, \bar{N})$ holds.

Proof. The "only if" direction follows directly from Theorem 5.1 Now we prove the "if" direction. Suppose $\operatorname{BiREG}_{C, D}(\bar{M}, \bar{N})$ holds. By Theorem [5.1, there exists a $(C, D)$-biregular graph $G=$ $\left(U, V, E_{1}, \ldots, E_{\ell}\right)$ of size $(\bar{M}, \bar{N})$. This graph $G$ is not necessarily complete. So suppose $U=$ $U_{1} \cup \cdots \cup U_{m}$ and $V=V_{1} \cup \cdots \cup V_{n}$ is the witness of the $(C, D)$-biregularity. If $G$ is not complete, then we perform the following. For every $u \in U$ and $v \in V$ such that $(u, v) \notin E_{1} \cup \cdots \cup E_{\ell}$, we do the following.

- Let $u \in U_{i}$ and $v \in V_{j}$.

- Pick an index $l \in\{1, \ldots, k\}$ such that $C_{l, i}, D_{l, j} \in \mathbb{N}$.

(Such an index $l$ exists since $(C, D)$ is an easy pair.)

- Connect $u$ and $v$ with an $E_{l}$-edge.

The resulting graph is now complete and still $(C, D)$-biregular. This completes our proof of Lemma 7.10 .

If $(C, D)$ is not an easy pair, then by Lemma 7.9 , the values in the entries (in $\bar{X}$ and $\bar{Y}$ ) corresponding to the columns in $C$ and $D$ that make them not an easy pair must be bounded. These values can be encoded as partial graphs as described in the previous section. This completes our proof of Theorem 5.2 .

\section{Proof of Theorem 5.4}

The proof is by observing that the existence of a $(C, D)$-directed-regular graph of size $\bar{N}$ is equivalent to the existence of a $(C, D)$-biregular graph of size $(\bar{N}, \bar{N})$. We explain it more precisely below.

- Suppose $G=\left(V, E_{1}, \ldots, E_{\ell}\right)$ is a $(C, D)$-directed-regular graph of size $\bar{N}$.

Then, for every vertex $v \in V$, we "split" it into two vertices $u$ and $w$ such that $u$ is only adjacent to the incoming edges of $v$ and $w$ to the outgoing edges of $v$. See the illustration below. The left-hand side shows the vertex $v$ before the splitting, and the right-hand side shows the vertices $u$ and $w$ after the splitting.
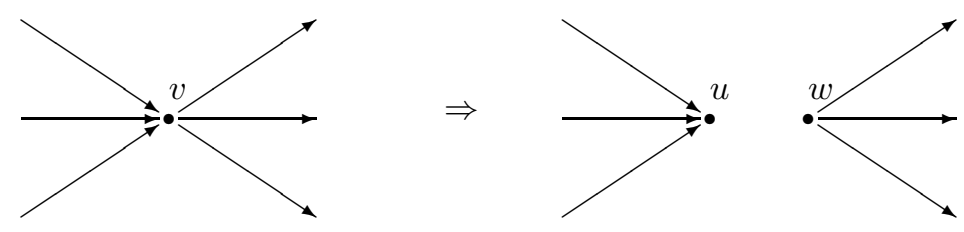

Let $U$ be the set of vertices $u$ 's and $W$ the set of vertices $w$ 's after splitting all the vertices in $V$. Ignoring the orientation of the edges, the resulting graph is a bipartite graph with vertices $U \cup W$ and it is a $(C, D)$-biregular graph of size $(\bar{N}, \bar{N})$.

- Suppose $G=\left(U, W, E_{1}, \ldots, E_{\ell}\right)$ is a $(C, D)$-biregular graph of size $(\bar{N}, \bar{N})$. Let $U=U_{1} \cup$ $\cdots \cup U_{m}$ and $W=W_{1} \cup \cdots \cup W_{m}$ be the witness of the $(C, D)$-biregularity. We denote by $U_{i}=\left\{u_{i, 1}, \ldots, u_{i, K_{i}}\right\}$ and $W_{i}=\left\{w_{i, 1}, \ldots, w_{i, K_{i}}\right\}$ for each $i=1, \ldots, m$.

Now we put the orientation on all the edges from $U$ to $W$. Then we merge every two vertices $u_{i, j}$ and $w_{i, j}$ into one vertex $v_{i, j}$. This way, we obtain a $(C, D)$-directed-regular graph $G=$ $\left(V, E_{1}, \ldots, E_{\ell}\right)$ with $V=V_{1} \cup \cdots \cup V_{m}$ be the witness of the $(C, D)$-regularity where $V_{i}=$ $\left\{v_{i, 1}, \ldots, v_{i, K_{i}}\right\}$ for each $i=1, \ldots, m$. 
However, with such merging it is possible that there is a self-loop $(v, v)$ in $G$ or a pair of edges $\left(v, v^{\prime}\right),\left(v^{\prime}, v\right) \in E_{1} \cup \cdots \cup E_{\ell}$. We can get rid of the self-loop $(v, v)$ without violating the $(C, D)$ directed-regularity as follows. The trick is similar to the one used before. Assuming that the size of each $\left|V_{1}\right|, \ldots,\left|V_{m}\right|$ is big enough and there are enough edges in each $E_{1}, \ldots, E_{\ell}$, there is an edge $\left(v^{\prime}, v^{\prime \prime}\right)$ of the same type such that both $v^{\prime}, v^{\prime \prime}$ are not adjacent to $v$. Deleting the edge $(v, v)$ and $\left(v^{\prime}, v^{\prime \prime}\right)$, and adding the edges $\left(v^{\prime}, v\right)$ and $\left(v, v^{\prime \prime}\right)$, we obtain a $(C, D)$-directedregular graph with one less self-loop. We do this repeatedly until there is no more self-loop. See the illustration below.

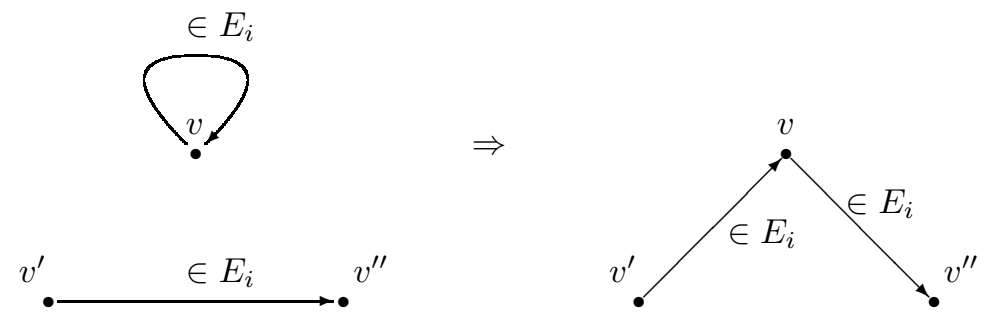

Similarly, we can get rid of a pair of edges $\left(v, v^{\prime}\right),\left(v^{\prime}, v\right) \in E_{1} \cup \cdots \cup E_{\ell}$ without violating the $(C, D)$-directed-regularity as follows. Again, the trick is similar to the one used before. Assuming that the size of each $\left|V_{1}\right|, \ldots,\left|V_{m}\right|$ is big enough and there are enough edges in each $E_{1}, \ldots, E_{\ell}$, there is an edge $\left(w, w^{\prime}\right)$ of the same type such that both $w, w^{\prime}$ are not adjacent to either $v$ or $v^{\prime}$. Deleting the edge $\left(v, v^{\prime}\right)$ and $\left(w, w^{\prime}\right)$, and adding the edges $\left(v, w^{\prime}\right)$ and $\left(w, v^{\prime}\right)$, we obtain a $(C, D)$-directed-regular graph with one less parallel edges. We do this repeatedly until there are no more parallel edges. See the illustration below.
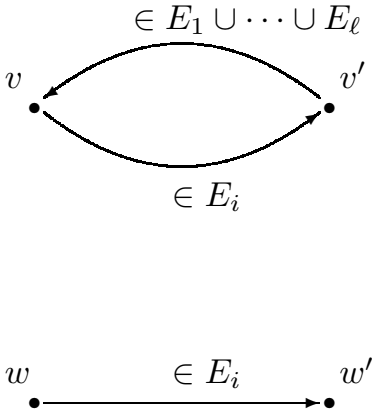

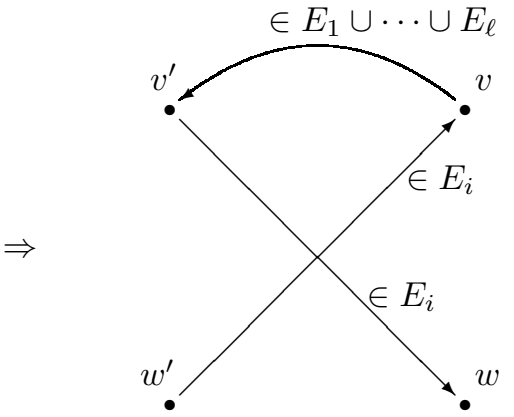

If some sets $V_{i}$ or $\left|E_{i}\right|$ are of a fixed size, those can be encoded in a partial graph in the same manner discussed in Subsection 7.4

We omit the technical details since we essentially run through the same arguments used in the previous section.

\section{Concluding remarks}

In this paper we have shown that the spectra of $C^{2}$ formulae are semilinear sets. The proof is by constructing the Presburger formulae that express precisely the spectra. As far as our knowledge is concerned, the logic $\mathrm{C}^{2}$ is the first logic whose spectra is closed under complement without any restriction on the vocabulary nor in the interpretation.

Furthermore, from our proof we can easily deduce a few easy corollaries. The first is that the family of models of a QMLC formula can be viewed as a collection of biregular graphs and regular digaphs in the following sense. Let $\phi$ be a QMLC formula and $\mathcal{R}=\left\{R_{1}, \ldots, R_{\ell}, \overleftarrow{R}_{1}, \ldots, \overleftarrow{R}_{\ell}\right\}$ be the 
set of binary relations used in $\phi$ and that $\overleftarrow{R}_{i}$ is the inversed relation of $R_{i}$. Let $K$ be the integer such that for all subformulae $\diamond_{R}^{l} \psi$ in $\phi$, we have $l \leq K$.

Let $\mathcal{T}_{\phi}$ is the set of all types in $\phi$. For a model $\mathfrak{A} \models \phi$, we partition $A=\bigcup_{T \in \mathcal{T}_{\phi}} A_{T}$, where $A_{T}=\{a \in A \mid a$ is of type $T\}$. That the model $\mathfrak{A}$ is a collection of regular graphs is in the following sense. Recall the matrices $D_{T}, \overleftarrow{D}_{T}, D_{S \rightarrow T}$ and $D_{T \rightarrow S}$ as defined in Section 6

For a type $T$, by restricting the relations $R_{1}, \ldots, R_{\ell}$ on the elements in $A_{T}$, we obtain a $\left(D_{T}, \overleftarrow{D}_{T}\right)$-regular digraph $G_{T}=\left(A_{T}, E_{1}, \ldots, E_{\ell}\right)$, where for each $E_{i}$,

$$
E_{i}=R_{i} \cap\left(A_{T} \times A_{T}\right)
$$

For two different types $S, T$, by restricting the relations on $A_{S} \times A_{T}$, we obtain a $\left(D_{S \rightarrow T}, \overleftarrow{D}_{S \rightarrow T}\right)$ biregular graph $G_{S, T}=\left(A_{S}, A_{T}, E_{1}, \ldots, E_{\ell}, E_{1}^{\prime}, \ldots, E_{\ell}^{\prime}\right)$, where each $E_{i}, E_{i}^{\prime}$ are

$$
E_{i}=R_{i} \cap\left(A_{S} \times A_{T}\right) \quad \text { and } \quad E_{i}^{\prime}=\overleftarrow{R}_{i} \cap\left(A_{S} \times A_{T}\right)
$$

Theorem 2.1 can be further generalised as follows. Let $\mathcal{P}=\left(P_{1}, \ldots, P_{l}\right)$, where $P_{1}, P_{2}, \ldots, P_{l}$ are unary predicates. Define the image of a structure $\mathfrak{A}$ as $\operatorname{Image}_{\mathcal{P}}(\mathfrak{A})=\left(\left|P_{1}^{\mathfrak{A}}\right|, \ldots,\left|P_{l}^{\mathfrak{A}}\right|\right)$. We also define the image of a formula $\varphi$ with predicates from $\mathcal{P}$ as $\operatorname{Image}_{\mathcal{P}}(\varphi)=\left\{\operatorname{Image}_{\mathcal{P}}(\mathfrak{A}) \mid \mathfrak{A} \models \varphi\right\}$. It must be noted here that $P_{1}^{\mathfrak{A}}, \ldots, P_{l}^{\mathfrak{A}}$ are not necessarily disjoint, and that they may not cover the whole domain $A$. For this reason, the notion of image is more general than the notion of manysorted spectrum which requires the unary predicates to partition the whole domain. With a slight adjustment in our proof in Section 6 we can obtain the following two corollaries.

Corollary 9.1 Let $\phi \in \mathrm{C}^{2}$ and $\mathcal{P}=\left(P_{1}, \ldots, P_{l}\right)$, where $P_{1}, \ldots, P_{l}$ be a set of unary predicates in $\phi$. The set $\left\{\operatorname{Image}_{\mathcal{P}}(\mathfrak{A}) \mid \mathfrak{A} \models \phi\right\}$ is semilinear.

Corollary 9.2 Let $\mathcal{P}=\left(P_{1}, \ldots, P_{l}\right)$. The following problem is decidable. Given a $\mathrm{C}^{2}$ formula $\phi$ and a Presburger formula $\Psi\left(x_{1}, \ldots, x_{l}\right)$, determine whether there exists a structure $\mathfrak{A} \models \phi$ such that $\Psi\left(\right.$ Image $\left._{\mathcal{P}}(\mathfrak{A})\right)$ holds.

There are still a few more questions that we would like to investigate for future work. The first natural question is: how can be $\mathrm{C}^{2}$ extended while keeping decidability? Using three variables $\left(\mathrm{FO}^{3}\right)$ one can easily encode a grid; therefore, the satisfiability problem is no longer decidable (and thus the image membership problem). However, we could extend $C^{2}$ by giving access to a relation having a property which is undefinable in $\mathrm{C}^{2}$, such as transitivity. In particular, $\mathrm{C}^{2}(<)$, that is, the logic $\mathrm{C}^{2}$ with access to a total order on the universe, seems powerful: Petri net reachability 29, 23, 24, reduces to image membership for $\mathrm{C}^{2}(<)$ formulae. We do not know whether a reduction exists in the other direction. Another possible extension is to add an equivalence relation to $C^{2}$.

\section{Acknowledgment}

We thank the anonymous referees for their comments and suggestions which greatly improve our paper. The second author also acknowledges the generous financial support of FWO, under the scheme FWO Pegasus Marie Curie fellowship.

\section{References}

[1] G. Asser. Das Repräsentenproblem in Prädikatenkalkül der ersten Stufe mit Identität. Zeitschrift für mathematische Logik und Grundlagen der Mathematik, 1: 252-263 (1955).

[2] A. Durand, N. D. Jones, J. A. Makowsky, M. More. Fifty years of the spectrum problem: survey and new results. Bulletin of Symbolic Logic 18(4): 505-553 (2012).

[3] H.-D. Ebbinghaus, J. Flum. Finite model theory. Perspectives in Mathematical Logic, Springer 1995. 
[4] R. Fagin. Contributions to the model theory of finite structures. Ph.D. thesis, University of California, Berkeley, 1973.

[5] R. Fagin. Finite model theory-a personal perspective. Theoretical Computer Science 116: 3-31 (1993).

[6] E. Fischer, J. A. Makowsky. On spectra of sentences of monadic second order logic with counting. Journal of Symbolic Logic 69(3):617-640 (2004).

[7] S. Ginsburg, E. H. Spanier. Semigroups, Presburger Formulas, and Languages. Pacific Journal of Mathematics 16:285-296 (1966).

[8] É. Grandjean. The spectra of first-order sentences and computational complexity. SIAM Journal on Computing 13(2): 356-373 (1984).

[9] É. Grandjean. Universal quantifiers and time complexity of random access machines. Mathematical Systems Theory 18(2): 171-187 (1985).

[10] É. Grandjean. First-order spectra with one variable. Journal of Computer and System Sciences 40(2): 136-153 (1990).

[11] E. Grädel. Why are Modal Logics so Robustly Decidable? Current Trends in Theoretical Computer Science 2001.

[12] E. Grädel, P. G. Kolaitis, M. Y. Vardi. On the Decision Problem for Two-Variable First-Order Logic. The Bulletin of Symbolic Logic, 3(1): 53-69 (1997).

[13] E. Grädel, M. Otto. On Logics with Two Variables. Theoretical Computer Science 224(1-2): 73-113 (1999).

[14] E. Grädel, M. Otto, E. Rosen. Two-Variable Logic with Counting is Decidable. In LICS 1997.

[15] M. Grohe, J. A. Makowsky, Editors. Model Theoretic Methods in Finite Combinatorics. Contemporary Mathematics 558, American Mathematical Society, 2011.

[16] Y. Gurevich and S. Shelah. Spectra of monadic second-order formulas with one unary function. In LICS 2003.

[17] S. Hakimi. On realizability of a set of integers as degrees of the vertices of a linear graph. I. Journal of the Soc. Indust. Appl. Math. 10(3): 496-506 (1962).

[18] I. Pratt-Hartmann. Complexity of the Two-Variable Fragment with Counting Quantifiers. Journal of Logic, Language and Information 14(3): 369-395 (2005).

[19] A. Hunter. Spectrum hierarchies and subdiagonal functions. In LICS 2003.

[20] N. Immerman. Descriptive complexity. Graduate texts in computer science, Springer 1999.

[21] N. Jones, A. Selman. Turing machines and the spectra of first-order formulas. The Journal of Symbolic Logic, 39: 139-150 (1974). (Also in STOC 1972.)

[22] H. Landau. On dominance relations and the structure of animal societies. III. The condition for a score structure. Bulletin of Mathematical Biophysics 15(2): 143-148 (1953).

[23] S. Kosaraju. Decability of reachability in vector addition systems. In STOC 1982.

[24] J. Leroux. The General Vector Addition System Reachability Problem by Presburger Inductive Invariants. In LICS 2009.

[25] E. Kieronski, J. Michaliszyn, I. Pratt-Hartmann, L. Tendera. Two-Variable First-Order Logic with Equivalence Closure. In LICS 2012 
[26] L. Libkin. Elements of Finite Model Theory. Springer 2004.

[27] J. Lynch. Complexity classes and theories of finite models. Mathematical Systems Theory 15(2): 127-144 (1982).

[28] C. Lutz, U. Sattler, F. Wolter. Modal logic and the two-variable fragment. In CSL 2001.

[29] E. Mayr. An algorithm for the general petri net reachability problem. In STOC 1981.

[30] M. Mortimer. On languages with two variables. Mathematical Logic Quarterly, 21(1): 135-140 (1975).

[31] M. Otto. Bounded Variable Logics and Counting - A Study in Finite Models Lecture Notes in Logic volume 9, Springer 1997.

[32] L. Pacholski, W. Szwast, L. Tendera. Complexity Results for First-Order Two-Variable Logic with Counting. SIAM Journal on Computing 29(4): 1083-1117 (2000).

[33] D. Rödding, H. Schwichtenberg. Bemerkungen zum Spektralproblem. Zeitschrift für mathematische Logik und Grundlagen der Mathematik, 18: 1-12 (1972).

[34] H. Scholz. Ein ungelöstes Problem in der symbolischen Logic. The journal of Symbolic Logic, 17: 160 (1952).

[35] W. Szwast, L. Tendera. FO ${ }^{2}$ with one transitive relation is decidable. In STACS 2013.

[36] K. N. Verma, H. Seidl, T. Schwentick. On the Complexity of Equational Horn Clauses. In CADE 2005.

[37] M. Vardi. Why is Modal Logic So Robustly Decidable? In Descriptive Complexity and Finite Models 1996.

[38] A. Woods. Some Problems in Logic and Number Theory and their Connections. Ph.D. thesis, University of Manchester, 1981.

[39] C. Wrathall. Rudimentary Predicates and Relative Computation. In SIAM Journal on Computing 7(2): 194-209 (1978). 